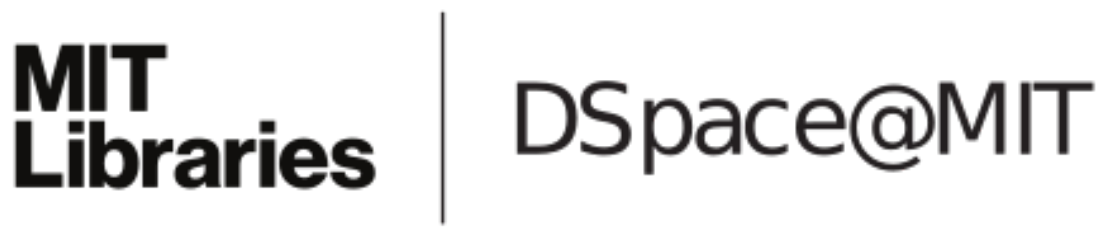

\author{
MIT Open Access Articles
}

\begin{abstract}
Validation of genetic algorithm-based optimal sampling for ocean data assimilation
\end{abstract}

The MIT Faculty has made this article openly available. Please share how this access benefits you. Your story matters.

Citation: Heaney, Kevin D. et al. "Validation of Genetic Algorithm-Based Optimal Sampling for Ocean Data Assimilation." Ocean Dynamics 66.10 (2016): 1209-1229.

As Published: http://dx.doi.org/10.1007/s10236-016-0976-5

Publisher: Springer Berlin Heidelberg

Persistent URL: http://hdl.handle.net/1721.1/106031

Version: Author's final manuscript: final author's manuscript post peer review, without publisher's formatting or copy editing

Terms of use: Creative Commons Attribution-Noncommercial-Share Alike 


\title{
Validation of Genetic Algorithm Based Optimal Sampling for Ocean Data Assimilation
}

\author{
Kevin D. Heaney, Pierre F. J. Lermusiaux, Timothy F. Duda, and Patrick J. Haley, Jr.
}

\begin{abstract}
Regional ocean models are capable of forecasting conditions for usefully long intervals of time (days) provided that initial and ongoing conditions can be measured. In resource-limited circumstances, the placement of sensors in optimal locations is essential. Here, a nonlinear optimization approach to determine optimal adaptive sampling that uses the Genetic Algorithm (GA) method is presented. The method determines sampling strategies that minimize a user-defined physics-based cost function. The method is evaluated using identical twin experiments, comparing hindcasts from an ensemble of simulations that assimilate data selected using the GA adaptive sampling and other methods. For skill metrics, we employ the reduction of the ensemble root-mean-square-error (RMSE) between the "true" data-assimilative ocean simulation and the different ensembles of data-assimilative hindcasts. A 5-glider optimal sampling study is set up for a $400 \mathrm{~km} x$ $400 \mathrm{~km}$ domain in the Middle Atlantic Bight region, along the New Jersey shelf-break. Results are compared for several ocean and atmospheric forcing conditions.
\end{abstract}

Index Terms- Genetic algorithms, ocean technology, optimization methods sampling methods, adaptive sampling, computational ocean modeling, data assimilation, error subspace statistical estimation, OSSE

\section{INTRODUCTION}

$\mathrm{M}$ ODERN dynamical ocean models, with suitable initial and boundary condition measurements, have a demonstrated capability to produce accurate forecasts over several days (Robinson, et al. 2006; Ramp et al, 2009, 2011; Haley et al, 2009; Lermusiaux et al, 2011; Hoteit et al. 2013). In order to maintain forecast capability by accounting for errors in the initial and boundary conditions, as well as errors within the scales not resolved by the computational model, a continuous set of measurements must be taken. The technique of measuring the ocean and integrating these measurements is termed dataassimilation and has been shown to be successful (e.g., Robinson et al., 1998; Lermusiaux et al., 2006b; Edwards, al. 2015). The challenge facing ocean forecasters is that ocean sampling will always be resource limited. An approach is needed to determine the places within the ocean volume where measurements will produce the best ocean estimate or forecast. The combination of ocean forecasts, sampling network planning using model feedback, and data-assimilation is referred to as optimal adaptive sampling. This paper presents a method for optimal sampling of oceanographic features for data assimilation. A quantitative validation of the method's ability to find an optimal sampling strategy is made. The focus of the methodology is to generate a set of targeted observations, which are consistent with a user-defined cost function, permitting the weighting of specific oceanographic dynamics, spatial variability and model uncertainty. The quality of the targeted observations is evaluated using identical twin experiments, similar to the approach of (Hamill, et al. 2013) where a meteorological numerical assimilation experiment using targeted Winter Storm Reconnaissance (WSR) data was conducted to evaluate the value added.

There has been substantial work in the field of path planning and adaptive sampling in the past decade, particularly with the development of autonomous underwater vehicles (AUVs) for ocean data collection. For general reviews on oceanic path planning, we refer to (Lolla, 2012; Lolla et al. 2014a; Lermusiaux et al, 2016) and for general reviews on oceanic adaptive sampling to (Curtin et al. 1993; Leonard et al. 2007; Lermusiaux, 2007; Roy et al, 2007). Recent efforts for autonomous adaptive sampling include: adaptive sampling via Error Subspace Statistical Estimation (ESSE) with non-linear predictions of error reductions (Lermusiaux 2007); control of coordinated patterns for ocean sampling (Zhang et al, 2007); a mathematical approach to optimally sampling targeted environmental hotspots in the 'MASP uncertainty framework' or multi-robot adaptive sampling problem (Low, et al. 2013); Mixed Integer Linear Programming (MILP) for optimal-sampling path planning (Yilmaz et al. 2008); nonlinear optimal-sampling path planning using genetic algorithms (Heaney, et al. 2007); dynamic programming and

Kevin D. Heaney is with the Ocean Acoustical Services and Instrumentation Systems Inc. Fairfax Station, VA 22039 USA (heaney@oasislex.com). Pierre F. J. Lermusiaux and Patrick Haley are with Massachusetts Institute of Technology, Cambridge MA, USA (pierrel@mit.edu, phaley@mit.edu)

Timothy F. Duda is with the Woods Hole Oceanographic Institution, Woods Hole MA, USA (tduda@whoi.edu). 
onboard routing for optimal-sampling path planning (Wang, et al. 2009); command and control of surface kayaks over the Web, directly read from model instructions (Xu et al., 2008); automated sensor networks aiming to facilitate ocean scientific studies (Schofield et al, 2010), and optimal design of glider-sampling networks (Alvarez and Mourre, 2012; Ferri et al, 2015). Many adaptive sampling efforts involve feature-based sensing, whereas others involve statistically measured improvements in the multi scale model fields. Oceanographic uncertainty, from model covariance estimates, can be used to help define the search strategy. Frolov et. al. (2014) used the best linear unbiased estimator (BLUE) algorithm to build the search paths for both a set of spacefilling algorithms (a "stretched lawnmower" and an A* algorithm) and a non-linear genetic algorithm (GA). Application to a simulated autonomous sampling network addressing distribution of phytoplankton off the central coast of California, quantitatively demonstrated that the optimal sampling approach (GA) outperformed the space-filling algorithms because of its ability to revisit areas of higher uncertainty. Adaptive sampling using clustering techniques have been applied to the adaptive sampling problem where covariance information was unavailable, but the field variance can be used (Cococcioni, et al. 2015). This clustering approach has the advantage of being computationally efficient and permits the combination of maneuverable and non-maneuverable assets. In addition to model-based adaptive sampling used in a mission-planning mode, adaptation directly from the sampled data can be performed. A front following technique (Petillo, et al. 2015) was developed to autonomously direct a vehicle in 2 and 3 dimensions to follow an isotherm associated with an oceanographic front directly from the observations of a CTD sensor on the vehicle. Other observation targeting techniques used in meteorology and weather forecasting (Berliner et al. 1999) include schemes based on the ensemble transform methods and Gaussian updates (Bishop, et al. 2001; Majumdar et al. 2002) as well as breeding methods, either in the full state-space (Toth and Kalnay 1997, Lorenz and Emanuel 1998) or in an unstable subspace (Carrassi, et al. 2007; Lermusiaux et al, 2007).

Intuitively we expect that placing sensors in regions where there is significant model uncertainty (Lermusiaux 2006, Lermusiaux, Chiu et al. 2006a), forecast sensitivity to current conditions, strong ocean dynamics such as fronts (Ogren, Fiorelli et al. 2004, Leonard, Paley et al. 2007; Zhang et al, 2012), or acoustic sensitivity would be advantageous. The approach presented here utilizes the genetic algorithm (GA) iterative search method to determine optimal placement of sensor suites within ocean fields produced by ocean forecast systems. Here, optimal means the spatial-temporal sampling scheme which minimizes a user-defined cost function, typically a combination of model variability, ocean dynamics, and acoustic sensitivity. A more formal notion of optimality would involve identification of a sampling scheme that brings a forecast model output the closest to reality as possible, given the sensor assets. This is not done here, as it involves an additional level of complexity (e.g. Lermusiaux et al., 2016; Lolla, 2016) to an adaptive sampling cost function that is already complex. For similar reasons, but also because our planning duration will be limited to a few days, we will not consider the effects of ocean currents in planning the path of vehicles. For such effects of currents, we refer to (Lolla et al, 2014a,b, 2015; Subramani et al 2015, Subramani and Lermusiaux, 2016) The present genetic-algorithm procedure was originally presented in (Heaney and Duda 2006; Heaney, Gawarkiewicz et al. 2007), but was not evaluated within a multiscale ocean environment and in a systematic fashion, using a statistical ensemble of simulations. In this paper, the same approach is applied to an ocean environment that includes multiscale dynamics, from internal tides to mesoscale and larger scale dynamics. The ocean region is around the Shallow Water 2006 (SW06) experiment area, within the Middle Atlantic Bight shelfbreak front region, near the Hudson Canyon. The genetic-algorithm approach itself is also extended to multiple ocean sensing platforms and to more complex cost functions that combine diverse objective functions, so as to illustrate the versatility of the genetic-algorithm. A critical investigation is a quantitative analysis of the solution behavior (convergence and uniqueness) and a statistical validation of the performance of the adaptive sampling plan based on an ensemble of simulations that assimilate the optimally sampled data (instead of a single simulation that assimilates this data). As part of the latter statistical Observation System Simulation Experiments (statistical OSSEs), the best and mean performances of the genetic-algorithm sampling are compared to these of two other sampling schemes, a random-sampling plan and a classic gridded-sampling plan. Two dynamical regimes are also considered in these OSSEs, the regimes before and after the passage of a Tropical storm over the region. A final difference is that the benchmark "True" ocean state estimate is a multiscale reanalysis from a realistic two-way nested data-assimilative ocean simulation (e.g. Haley and Lermusiaux, 2010) that was validated by comparisons with independent observations.

The paper is organized as follows. In Section II the Shallow Water 2006 experiment and ocean model are presented. The genetic algorithm (GA) based optimization method is presented in Section III. The behavior of the solution for a single platform and for a 5-glider sampling scheme is presented in Section IV. The validation of the approach is presented in Section V. A comparison of the GA solution with random sampling solutions and an oceanographically relevant sampling strategy is completed. For quantitative comparisons, a 'shooting method' and a full Observing System Simulation Experiment (OSSE) are employed. For the OSSE, observables (temperature/salinity) from the GA selected paths are assimilated into an independent ocean ensemble simulation. Section VI contains the summary and conclusion. 


\section{Shallow WATER 2006 EXPERIMENT AND OCEAN MOdeL}

The Office of Naval Research sponsored Shallow Water 2006 (SW06) experiment included collection of a large set of oceanographic and acoustic measurements on the New Jersey Shelf/Hudson Canyon region of the Mid-Atlantic bight (Tang, Moum et al. 2007, Chapman and Lynch 2010, Lin, Newhall et al. 2010). The operation took place in late July-September 2006. The effort included moored and shipboard physical oceanographic observations. Data-driven ocean modeling associated with the project was done with a primitive equation (PE) ocean model of the Multidisciplinary Simulation, Estimation and Assimilation System (MSEAS Group, 2013). The MSEAS-PE modeling was adopted to create the synthetic ocean for this study.

Reanalysis physical fields of the SW06 operational area were created using the MSEAS free-surface PE (Figure 1). The MSEAS-PE was configured for implicit two-way nesting. The coarse domain was a $522 \mathrm{~km} \mathrm{x} 447 \mathrm{~km}$ domain, with $3 \mathrm{~km}$ resolution, to simulate the region of influence. The fine domain was a $172 \mathrm{~km} \times 155 \mathrm{~km}$ domain, with $1 \mathrm{~km}$ resolution, to refine the simulated dynamics in the main acoustic test region just south of the Hudson Canyon. This site was chosen due to the complex bathymetry and oceanography of the shelf-break environment. Both domains employed 30 terrain-following vertical levels in a double-sigma configuration (Haley and Lermusiaux 2010). The bathymetry used was a combination of the NOAA Coastal Relief Model (NOAA 2006) combined with V8.2 (2000) of the Smith and Sandwell topography(Smith and Sandwell 1994) in the deep regions. The estimation of the initial conditions was based on two objective analyses, one inshore and one offshore of the expected shelfbreak front, using both in situ synoptic data (Rutgers SeaGliders, NMFS cruises, CTD casts collected aboard the research vessels Knorr, Quest and Tioga, as well as Scanfish data) and historical data (NMFS, World Ocean Data Base, Gulf Stream Feature analyses, Buoy data, etc.). These two analyses were combined using a shelfbreak front feature model (Sloan 1996, Lermusiaux and Robinson 1999, Gangopadhyay, Robinson et al. 2003). The Gulf Stream was initialized based on historical CTD profiles and estimates of its position based on SST and NAVOCEANO feature analyses. Dynamically balanced initial velocity fields were constructed from the mapped temperature/salinity (T/S) fields, reduced dynamics and kinematic constraints at the coasts (Haley, Agarwal et al. 2015). The simulations were forced with atmospheric fluxes derived from weather research and forecasting (WRF) model simulations (J. Evans, personal communication) and FNMOC (The US Navy Fleet Numerical Meteorology and Oceanography Center), and laterally forced with linear barotropic tides (Egbert and Erofeeva 2002, Logutov and Lermusiaux 2008). Twice-daily assimilation of the synoptic data was applied to control uncertainties. SST was also assimilated on relatively cloud-free days (Aug 14, 17, 19, 21, 23, 25).

a)

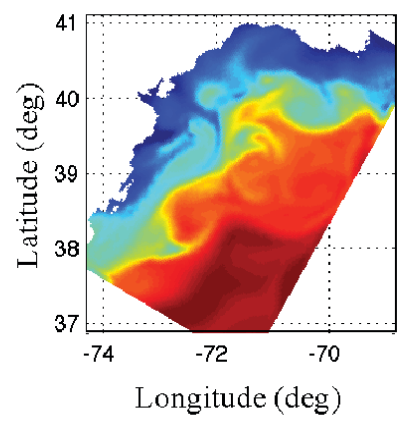

C)

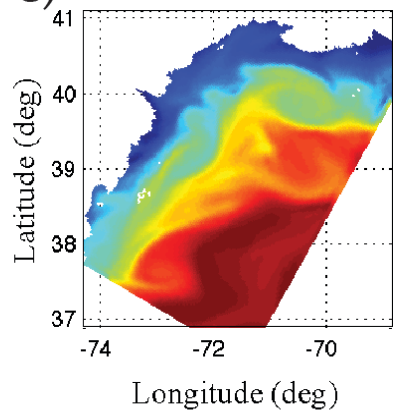

b)
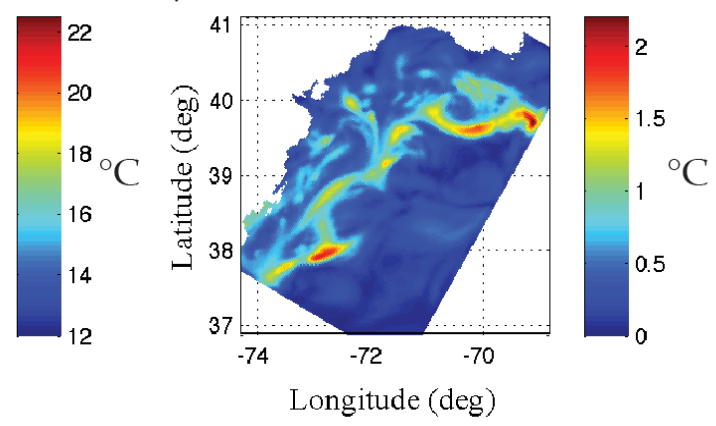

d)

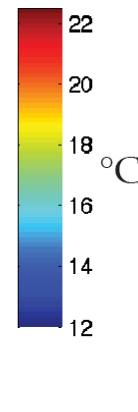

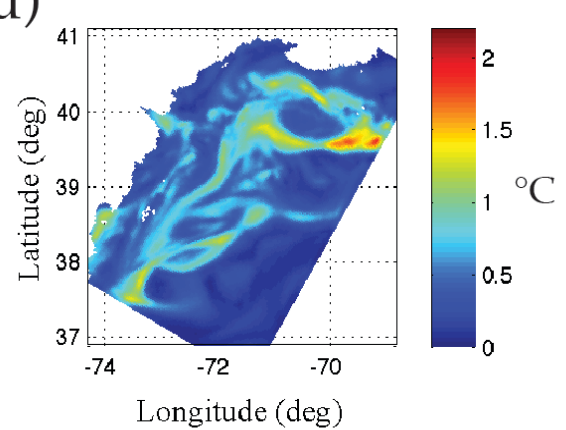

Figure 1. Reanalysis fields computed using the MSEAS primitive-equation ocean model and the Error Subspace Statistical Estimation (ESSE) scheme for the SW06 region. a) August 24-27 ensemble mean of the temperature ( $T$ along the $24.7 \mathrm{~g} / \mathrm{cc}$ isopycnal). b) August $24-27$ ensemble standard deviation of the $T$. c) Sep 4-7 ensemble mean of the $T$ (along the $24.7 \mathrm{~g} / \mathrm{cc}$ isopycnal). d) Sep 4-7 ensemble standard deviation of the $T$. Each panel is the temporal average over the modeled time period of 48 hours. 
As part of the MSEAS reanalysis fields, an ensemble of 17 simulations was constructed using different perturbations of initial conditions and model parameters using the Error Subspace Statistical Estimation (ESSE) schemes (Lermusiaux 2004, Lermusiaux 2006, Lermusiaux, Chiu et al. 2006a) and representing expected uncertainties including unknown variability. Two distinctive periods were selected to investigate how optimal sampling paths vary due to a change in the mean ocean state, in response to the passage of a strong storm. The first period is 24-27 Aug 2006, prior to the passage of tropical storm Ernesto. The second is 4-7 Sep 2006, after the passage of Ernesto. Using these ensembles of simulations, four different classes of sampling strategies were generated and compared twice, once for each of the two periods (Section V). Each of these sampling strategies simulated a fleet of 5 gliders operating for 2 days in the shelfbreak region. The ensemble average and standard deviation of the temperature along the isopycnal surface $\sigma=24.7 \mathrm{~g} / \mathrm{cc}$ surface for the two periods are shown in Figure 1.

Although appreciable efforts were made to bring the model ocean fields as close as possible to the true ocean state at the time of the SW06 field effort, the fidelity of the model ocean fields is not essential to this study. However, the good quality of the model fields gives some confidence that the evaluation of the genetic algorithm optimal sampling determination scheme (GAOSDS) applies to the real ocean, not simply to a specialized or idealized synthetic ocean field.

\section{OPTIMAL SAMPLING APPROACH}

In order to solve the sensor placement problem with a computational optimization algorithm, two sets of ancillary algorithms are required. The first maps a single point in the multi-dimensional search space to a platform laydown, defining start positions and times and subsequent turns for all vehicles. The second is a definition of the cost function that is used to evaluate the fitness or energy of each laydown. Section IIIA defines the search platform and Section IIIB the cost function. Section IIIC presents the Genetic Algorithm approach used to determine the optimal plan. Note that once the search plan mapping and cost functions have been defined, any numerical optimization algorithm can be applied to the chosen objectives. The two most common optimization schemes are Genetic Algorithms and Simulated Annealing. We also note that to handle conflicting objectives and cost functions, multi-objective optimization, linear or even nonlinear (e.g. Miettinen, 2012), can be employed.

\section{A. Individual Sensor Definition}

A platform is defined as any sensor system that can be deployed in the ocean and return data. Relevant ocean platforms are gliders, autonomous underwater vehicles (AUVs), thermistor moorings, acoustic measurement systems, buoys, shipboard tow-yo (Scanfish) and XBT measurements. The code which generates a platform sampling pattern must convert a vector of search values (genes) $\beta=\left[\begin{array}{ll}0 & 1\end{array}\right]$ (random with a uniform a-priori probability density function (pdf) in the first generation) to a position vs. time vector $\boldsymbol{r}(t)$. The simplest platform is a stationary mooring, which has no time-dependence and is simply defined as $r=$ $\left[\beta_{I} X \beta_{2} Y\right]$ where $X$ and $Y$ are the maximum dimensions of the domain in latitude/longitude. Ship-board measurements, which have high flexibility, can be broken into a set of straight survey lines with initial start positions and length and direction of each radial run.

The type of measurement considered here is a set of undulating autonomous sub-surface gliders. Each glider has an initial position $(x, y)$ and bearing. Each glider is deployed for 48 hours with a single turn at 24 hours. The turn is a random value with a uniform pdf between $-35^{\circ}$ and $35^{\circ}$. The limits are chosen to prevent the glider from turning back on itself and yield paths that are closer to straight, the traditional method of deploying gliders. The horizontal glider velocity through the water is $30 \mathrm{~cm} / \mathrm{s}$. The glider is subject to the local depth averaged time varying velocity vectors. Note that the depth dimension has been collapsed and a 2-dimensional search pattern is sought. For this particular implementation, the glider attempts to maintain a particular bearing by adaptively steering to maintain its preferred bearing. In some regions of the ocean, where currents are strong, it is not possible to maintain a particular heading with a glider. The current implementation of the GA does not include 3-dimensional tracks of the platforms, but simply inputs a min-max depth excursion for each platform. This sidesteps the important issue of sampling aliasing due to the slow vertical motions of the gliders. For the temporal and spatial scales resolved in current oceanographic models these aliasing issues are not significant. The inclusion of forecast currents in the generation of glider search paths is critical to the GA forming glider paths that are experimentally achievable.

\section{B. Cost Function Definition}

The general approach is to evaluate the cost function for a set of platform laydowns (glider sampling paths $\boldsymbol{r}_{t}(t)$ in the examples that follow). As defined below, the cost function has a scalar value for each platform laydown plan. Given that cost functions are scalars, multiple cost functions can be defined and added in a normalized weighted fashion. Each of these individual cost functions is termed a constituent cost function (CCF). The weighting vector $(W)$ determines the linear combination of constituent cost functions, termed the global cost function, which is to be minimized. 
Within this framework, the cost function incorporates what the user determines to be "optimal". Specifically in this paper we refer to optimal as: the platform laydown having the minimum user-defined cost function upon attainment of the numerical search exit criterion. It is up to the user to define a set of cost functions tailored to the ocean physics, model uncertainty measures, and other factors judged to be important. Quite regularly, the GA optimization (minimization) yields platform laydown patterns that sample the regions of the highest model uncertainty or most-active ocean dynamics, as desired. For more general cases, multi-objective optimization approaches (e.g. Miettinen, 2012; Collette and Siarry, 2013) can also be employed to merge different cost functions and objectives in a quantitative fashion.

In order to reduce the computational burden of cost function evaluation it is desirable to reduce the field from four dimensions to three by collapsing the depth spatial dimension $(z)$ and retaining lateral dimensions along with time $(t)$. In order to sample the ocean fields, several options are available including the depth average, the surface field values (e.g. temperature), an average of the surficial waters $(\mathrm{z}<50 \mathrm{~m})$, or the ocean field along a particular isopycnal (e.g., for temperature, $T_{\sigma}$ ). With an eye toward application of oceanographic forecasting in predictive acoustic computations, the density isopycnal of $\sigma=24.7 \mathrm{~g} / \mathrm{cc}$ is selected. Variations of temperature along this isopycnal indicate boundaries between shelf and slope waters in the Mid-Atlantic Bight region.

Two types of averages will be performed in the computation of cost functions: spatial averaging at sensor positions for moving sensors, $\langle\cdot\rangle_{p}$, and averaging over ensembles of model states, $\langle\cdot\rangle_{e}$.

1) Measured Temperature Spatial Variability $\left(F_{\sigma}\right)$

The first CCF is defined to capture to ocean spatial variability. The spatial variability cost function, is the negative of the standard deviation of the temperature on the isopycnal surface $\sigma=24.7 \mathrm{~g} / \mathrm{cc}$ measurements over the glider paths. Rather than averaging over ensembles, and thereby smoothing out the spatial features, the ensemble that is closest to the mean is selected. It is defined by

$$
F_{\sigma}=-\left\langle\left(T_{\sigma}(r)-\bar{T}_{\sigma}\right)\right\rangle_{p}^{1 / 2}
$$

where $T_{\sigma}$ is measured temperature along each path and $\bar{T}_{\sigma}=\left\langle T_{\sigma}\right\rangle_{p}$ is the mean sampled temperature of the individual platform. The cost function value for the platform laydown is the mean of the sensor $F_{\sigma}$ values over platforms. Note that the motion of the glider is taken into account, so that temperature values along a path are extracted from the forecast at sequential times. This cost function yields strong negative values in regions where there are fronts or other strongly range-dependent temperature fields. The philosophy is that measurements taken where the ocean is the most dynamic (on temporal-spatial scales defined by glider sampling) will produce the most improvements in model forecast skill. This cost function, labeled $F_{\sigma}$ for identification purposes throughout the paper, for regions where the oceanographic spatial scale lengths (Rossby radius) are larger than the glidersampling path, is effectively an edge (front) detector. In this paper the cost functions for each glider will be averaged, so the square root is taken before gliders are averaged. The temperature field $\mathrm{T}_{\sigma}$ along the chosen isopycnal is computed as a function of time and used as the input temperature field for the cost function computation.

2) Model Forecast Variability $\left(F_{f i}\right)$

The model uncertainty cost function is the standard deviation of the ensemble model temperature fields as a function of $x, y$, and $t$. The isopycnal $\sigma=24.7 \mathrm{~g} / \mathrm{cc}$ is used to collapse the depth dimension for each model forecast. The cost function is the negative mean of the model uncertainty field (ensemble standard deviation) computed along the trajectory of the glider. This is a normalized path integral through the model uncertainty space and will select glider paths that sample at regions where there is the most model variability. The mathematical expression for the $F_{f v}$ cost function for each platform path $(p)$ is:

$$
F_{f v}=\left\langle\left\langle\left(T_{\sigma}(r, t)-\left\langle T_{\sigma}(r, t)\right\rangle_{e}\right)^{2}\right\rangle_{e}\right\rangle_{p}
$$

\section{3) Distance potential (D)}

A constituent cost function that penalizes sample strategies that place multiple platforms within a particular temporal or spatial distance of each other is added to the oceanographic cost functions. This cost function, termed the distance potential (Leonard, Paley et al. 2007), uses two algebraic inverse functions with user specified temporal and spatial lengths: 


$$
D=\left\{\left(\frac{1}{\Delta r / \hat{r}-\alpha}\right)^{2}+1\right\}\left\{\left(\frac{1}{\Delta t / \hat{t}-\alpha}\right)^{2}+1\right\}-1
$$

where $\Delta r$ and $\Delta t$ are the spatial and temporal separation between all platform pairs, $\hat{r}$ and $\hat{t}$ are temporal and spatial correlation distances (10km and 24 hours in this case) and $\alpha$ is a numerical scaling factor $(\sim 0.001)$ controlling the slope of the function and preventing infinity when either $\Delta r$ or $\Delta t$ is zero. The cost function is defined to be very large for positions within a correlation time or length and zero for positions well outside of these lengths. For the simulations presented here, the spatial correlation length is taken to be $20 \mathrm{~km}$ (an estimate of the first baroclinic Rossby deformation radius) and the temporal correlation length is taken to be 12 hours. The distance potential cost function is effectively a constraint. The algebraic difficulty of parameterizing a constraint for integer programming approaches (Yilmaz, et al. 2008) is by-passed by the inclusion of a cost function. The numerical optimization scheme selected will eliminate platform paths that violate this constraint automatically. The addition of new sorts of operational constraints can be achieved by simply including a cost function that penalizes paths that violate this constraint. Such an operational constraint - minimum water depth of operation, is presented next.

\section{4) Boundary Cost Function (B)}

It is probable that there will be operational constraints associated with the deployment of sensing platforms. For gliders, this certainly includes a minimum water depth of operation. Other geographic boundaries can exist, such as international borders or water-space exclusion zones during exercises. Rather than implementing these constraints as complicated mathematical systems for the definition of glider paths, a boundary cost function is defined and solutions that have glider paths outside a preferred zone of operation are severely penalized with a large positive cost function constituent. The optimization approach will then automatically de-select (kill) these individuals. This comes at a small computational cost but is well worth the conceptual simplicity. For the simulations presented below, one boundary cost function is used, assigning a value of 100 to any glider sampling that has a glider in water shallower than 50m depth (this includes glider paths lying over land).

\section{5) Global Cost Function (E)}

The global scalar cost function is what the Genetic Algorithm seeks to minimize. In defining the "optimal" deployment configuration of this diverse suite of sensors, it is up to the user to provide weights for the linear combination of constituent cost functions. This permits the user to weigh ocean dynamics, ocean variability, or any other assortment of cost functions (such as acoustic sensitivity). The weighted, normalized cost function $E$ is computed as follows:

$$
E(\vec{r})=\sum_{i} \frac{W_{i} C_{i}(\vec{r})}{\sigma\left(C_{i}\right)}+W_{B} B(\vec{r})+W_{D} D(\vec{r})
$$

where $W_{i}$ are weights and $\sigma\left(C_{i}\right)$ is the standard deviation computed for each of the constituent cost functions $C_{i}$. The underlying principle is that for a specific survey plan, or sensor geometry, a variety of constituent functions $(C)$ can be used at the discretion of the user, each evaluated from the modeled values of the field. The user selects the relative weights $W_{i}$. Normalization of the various constituent cost functions is conducted by computing the spatial standard deviation of the estimated cost function morphology. Normalization by the standard deviation non-dimensionalizes the cost function and permits the combination of many different surfaces with possibly disparate scales. Each normalized cost function is a dimensionless, normalized scalar, permitting the user defined weighted sum to be the global cost function used for optimization. In Figure 2, the cost function morphology is estimated for the August 24-27 and September 4-7 time periods for each cost function, $F_{\sigma}, F_{v}$ and a normalized linear combination $\left(W_{i}=1\right.$, for $\left.i=1,2\right)$. To estimate the morphology, a brute-force mapping is performed. The region is divided into a subset (100x100) of uniformly spaced points. Two gliders are launched from each point. One travels due east for 24 hours, the other due north for 24 hours. Currents are neglected making these paths straight. The cost function is evaluated for each path and the minimum of the two is used as the estimate of the cost function morphology for that starting point. This morphology estimation is used only for computation of the dynamic range of each cost function and for plotting purposes. The optimization result is expected to outperform this search for a minimum value of the cost function. This modified brute-force mapping permits a qualitative understanding of the shape of the cost function, particularly with respect to initial launch position. A complete computation of the cost function, using 1 degree resolution in the bearing and 200x200 points in the horizontal for a glider that has an initial direction and a single turn would be $(200 \times 200 \times 360 \times 360)=4.8 \times 10^{9}$ computations per constituent cost function.

The cost functions plotted in Fig. 2 show how the ocean in this region is substantially different for the two time periods. Fig. 2 also illustrates both how different cost functions highlight different features and how the normalized combination can involve information from each cost function. 
a)

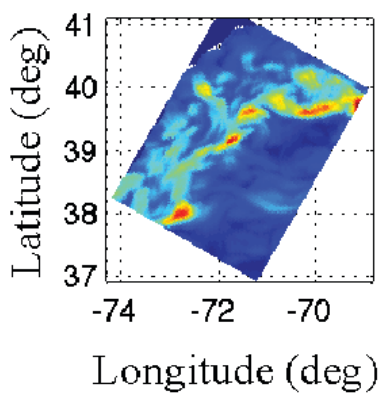

b)

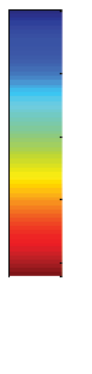

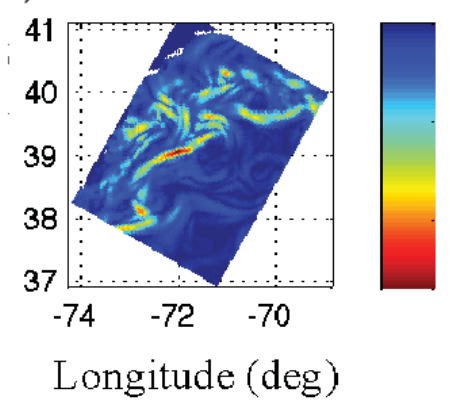

c)

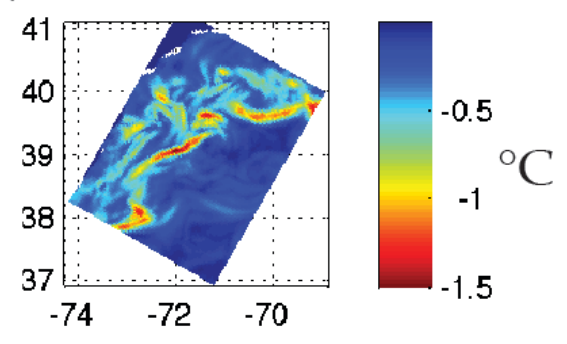

Longitude (deg) d)

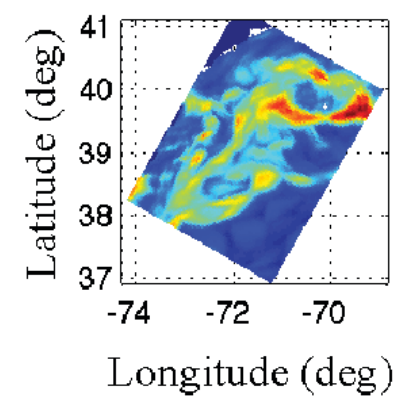

e)
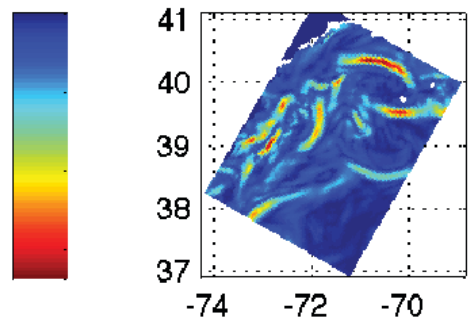

Longitude (deg) f)
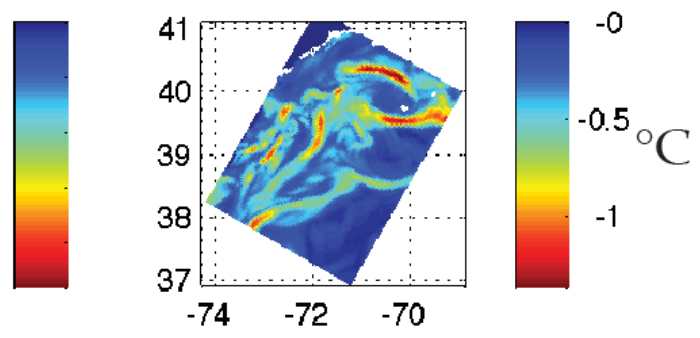

$-1$

Longitude (deg)

Figure 2. Estimated cost function morphology. The left column (panels a/d) is for the spatial variability of the measured $F_{\sigma}$, and is a measure of the standard deviation in space across the closest-to-mean ensemble $T_{\sigma}$ surface. The center column (panels b/e) is for model uncertainty $F_{f v}$, and is the time average of the ensemble variability of the $T_{\sigma}$ surface. The right column (panels $\mathrm{c} / \mathrm{f}$ ) is for the normalized (uniformly) weighted global cost function. Panels a-c (upper row) are for Aug 24-27 period, panels d-f (lower row) are for Sep 4-7.

\section{The Genetic Algorithm Technique}

The genetic algorithm (GA) is a standard searching technique for solving constrained large-dimensional non-linear optimization problems (Goldberg 1989). The GA has been successfully applied to many of these problems, including for example geo-acoustic inversion in underwater acoustics (Gerstoft 1994, Gerstoft and Gingras 1996). The algorithm is loosely based on the process of natural selection in evolutionary biology. A gene is defined as a vector that uniquely determines a parameter of the search space, such as sensor platform deployment coordinates. Based upon the analogy of natural selection a population is generated from a random sampling of a particular gene pool, which spans the multi-dimensional search space. A population is a set of individuals, each having a set of genes specifying a unique measurement plan, which we refer to as the sensor laydown. Thus, for the example five-glider problem to be outlined below, each individual represents a different time/space transect pattern. Beginning with an initial random sampling scheme (first generation), and iterating over generations, a cost function (or fitness) of each individual is computed. Using this information, fit individuals are selected and mated and a new generation of individuals is produced. Unfit individuals (those with poor cost function values) are not reproduced. A random crossover of parent genes generates the genes of the children. To reduce the probability of converging to a local cost function minimum, a small fraction of random mutations of individual genes are permitted for each generation. Reproduction and fitness testing occurs until an exit criterion is met. Example exit criteria are a minimum percentage change in the cost function (apparent convergence), or a maximum number of generations. 


\section{OPTIMIZATION CONVERGENCE AND UNIQUENESS}

\section{A. Single Platform Solutions}

In order to best examine the convergence and uniqueness of the optimization solutions, repeated searches were performed for a single-platform measurement system. The system was composed of a 48-hour deployment of one glider, with a single turn at the 24-hour mark. This gives four elements in the search vector (launch latitude, launch longitude, initial heading, heading after turn). The glider parameters are: horizontal speed $=30 \mathrm{~cm} / \mathrm{s}$, operating depth $0-600 \mathrm{~m}$ (or seafloor). The GA solution was computed for two time periods and two cost functions: $A u g-F_{\sigma} A u g-F_{f v}$, Sep- $F_{\sigma}$ Sep- $F_{f v}$. The GA parameters were: 100 individuals, 80 generations, $80 \%$ cross-over fraction, $3 \%$ mutation fraction, and the 4 best individuals were kept each generation. The GA was run with different random starting fields (with a uniform pdf) 20 times for each case. Sensitivity of the GA solution to specific parameter sets has been presented elsewhere(Gerstoft, Hodgkiss et al. 2003). The values of the best cost function value at each generation as a function of generation is plotted in Fig. 3 for each of the four cases. For each example, the boundary cost function $(B)$ is added to the constituent cost function. The distance potential cost function is not computed for a single platform $\left(W_{D}=0\right)$.

Fig. 3 shows that all of the single glider GA solutions converge by 40 generations (many by 20), The convergence to different values indicates that the search has settled in local minima of the cost function. For the $A u g-F_{\sigma}$ and $A u g-F_{f v}$ cases, there are clearly two clusters of cost function values, indicating clumpiness of the numerical values of the local minima that are capable of halting GA search evolution. The $\mathrm{Sep}-F_{\sigma}$ clearly converges to a tightly-limited range of cost function values for all 20 cases. The Sep- $F_{f v}$ example also converges in this way to a local minima, with the exception converging on the best solution. The slower rate of convergence of solutions for the $A u g-F_{f v}$ case compared with the $S e p-F_{f v}$ case is an indication of the comparatively complex morphology of the ocean in $A u g-F_{f v}$.

a)

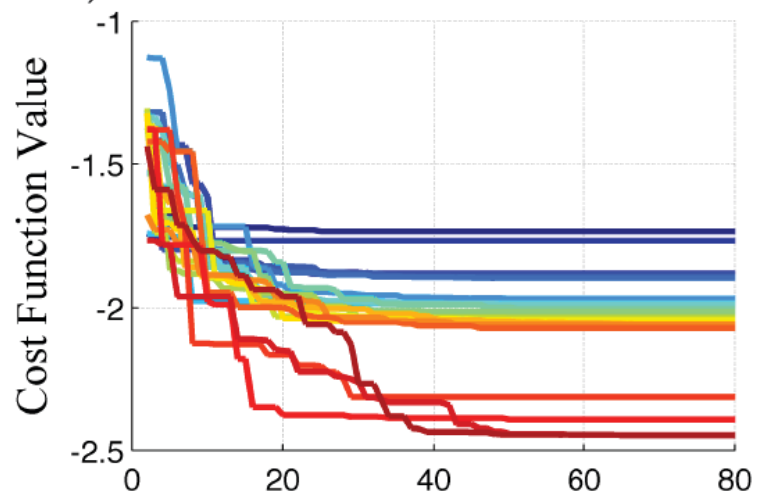

c)

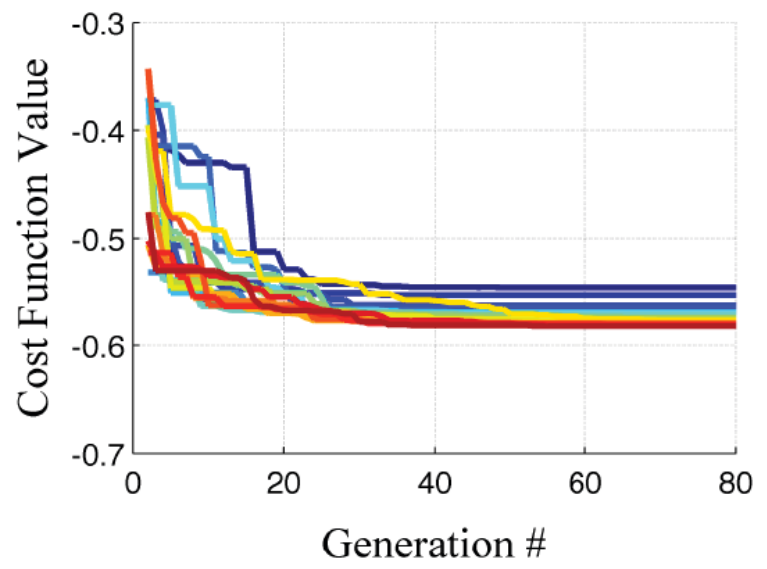

b)

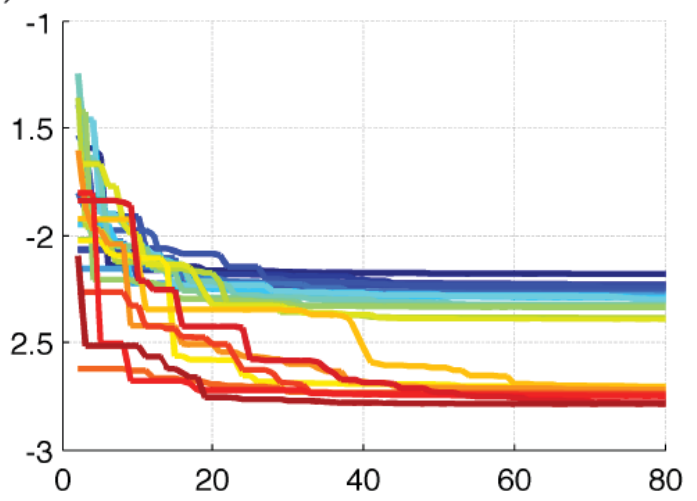

d)

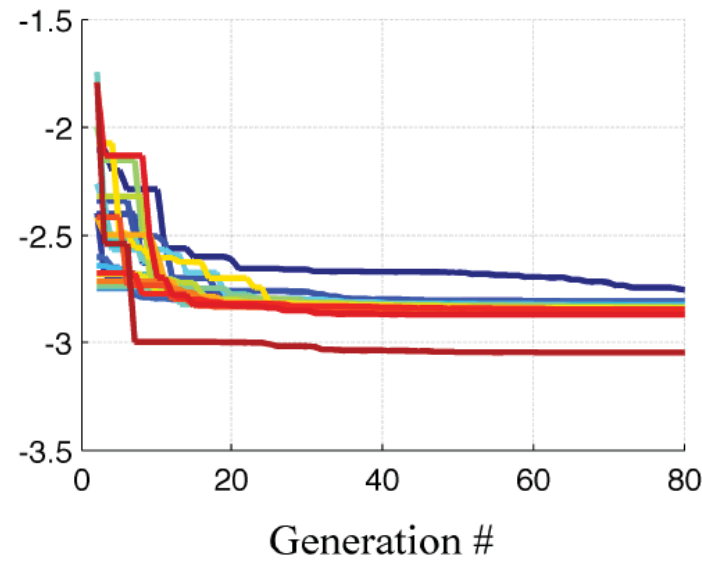

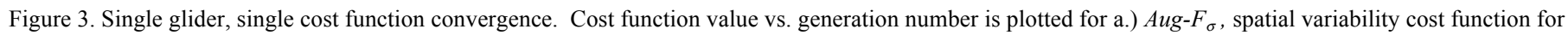
Aug. 24-27. b.) Aug- $F_{f v}$ Model uncertainty for Aug 24-27. c.) Sep- $F_{\sigma}$, spatial variability cost function for Sep. 4-7. d.) Sep- $F_{f v}$ Model uncertainty for Sep 4-7. The solutions are plotted in rank ordered color (red-blue) from best to worst. 
The solutions are plotted in Fig. 4 on top of the contour plots of the estimated cost function morphology. This provides insight into the uniqueness of the solutions. The colors of each solution are rank-ordered from best to worst (red to blue) as in Fig. 3 . Aug- $F_{\sigma}$ has clusters of solutions. The best solutions lie along the shelf-break in the southwest corner of the domain. Others lie in apparent local minima in the northeast. The $A u g-F_{f v}$ solution is spread amongst four minima, each with similar cost function values. For this cost function morphology (contoured), there is not a clear unique solution. The $S e p-F_{\sigma}$ solutions all lie within a global minimum in the northeast corner of the region. The Sep- $F_{f v}$ solutions are found in four different locations, with the best ones in the northernmost portion of the region. Note that all four cost functions produce optimal glider searches in different regions of the domain.

a)

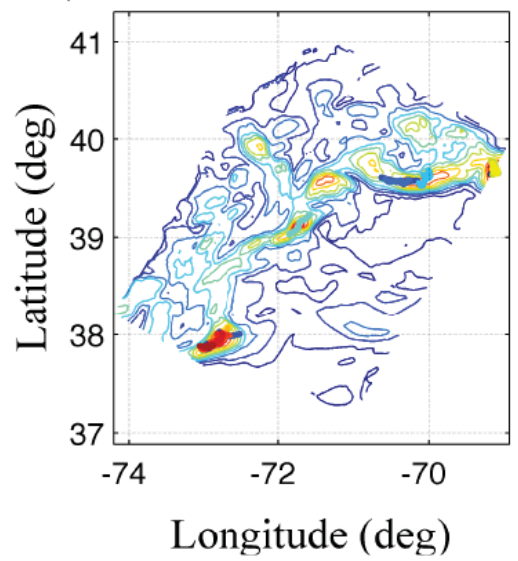

c)

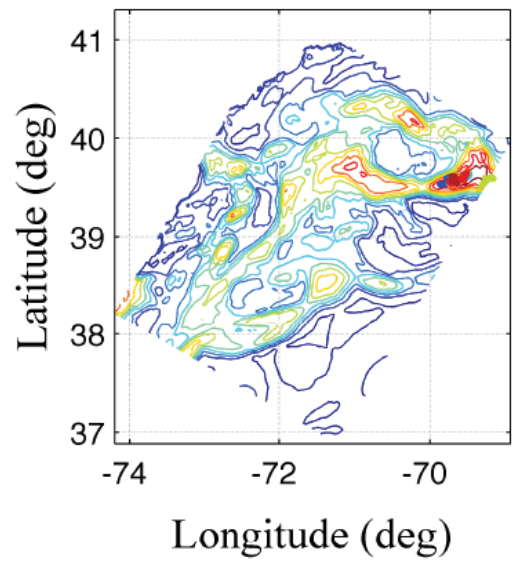

b)

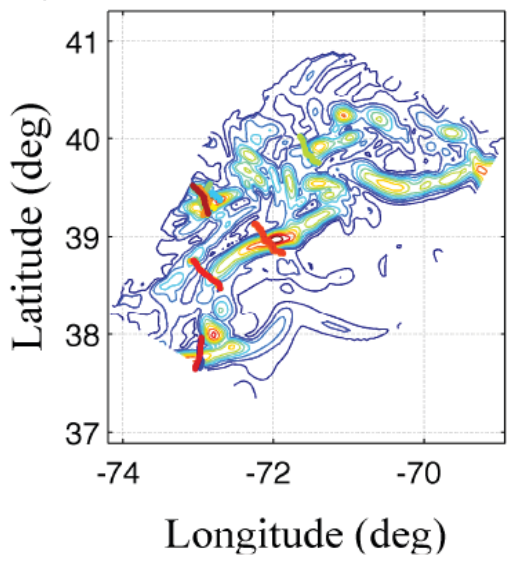

d)

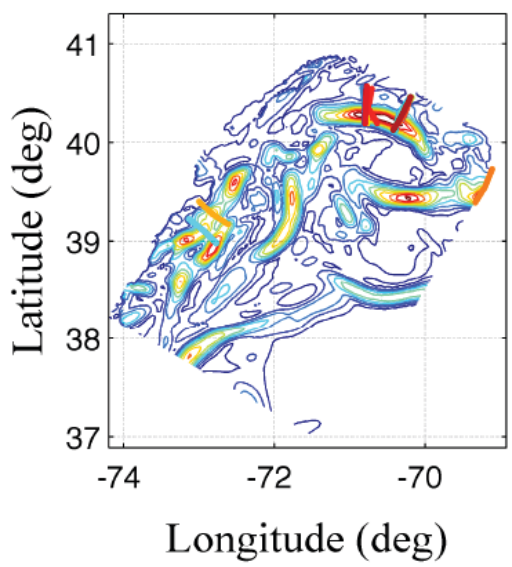

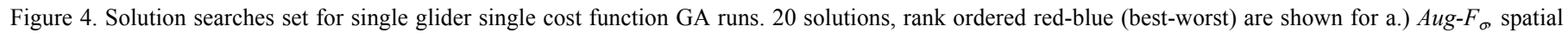

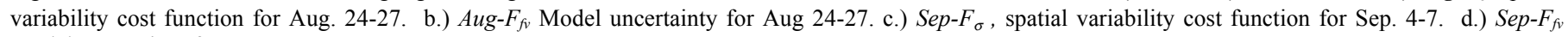
Model uncertainty for Sep 4-7.

\section{B. Multiple Platform Solutions}

Much of the utility of the GA solution approach outlined here lies in its ability to optimize the locations of multiple diverse platforms simultaneously. When multiple platforms are used, the convergence and uniqueness behavior of the GA solution differs from single-platform behavior. Whereas with a single platform the GA demonstrated a tendency to fall into a local minimum and thereby limit the final value of the cost function, the solution behavior with multiple platforms is for each platform to sample a different minimum. The distance-potential cost function prohibits two gliders from sampling the same local minima. In this section, the behavior of the GA solution for a five-glider optimization is presented. Each glider is deployed for 48 hours with a single turn at 24 . Thus there are 4 search parameters per glider, giving a search space with a dimensionality of 20 .

\section{1) Single Constituent Cost Functions}

The five-glider optimization was conducted using the spatial variability $\left(F_{\sigma}\right)$ and model uncertainty $\left(F_{f v}\right)$ cost functions 
separately for each of the time periods of interest $\left(A u g-F_{\sigma} A u g-F_{f v}, S e p-F_{\sigma} S e p-F_{f v}\right)$. For each cost function and time period, twenty GA solutions were computed. The parameters for the GA runs were the same as for the single-glider case with the exception of the maximum number of generations, raised to 140 for the five-glider case. The distance-potential constituent cost function $(D)$ is added to the $F_{f v}$ or $F_{\sigma}$ and the $B$ cost functions to prevent gliders from lying within a specified correlation time and space from one another. For brevity, the results for only two of the simulations, Aug- $F_{\sigma}$ and Sep- $F_{f v}$, are presented here. The others exhibit similar tendencies.

In Fig. 5, the results for the Aug- $F_{\sigma}$ cost function are presented. The upper left panel plots the cost function value vs. generation to display convergence. Note that the GA does not converge for this case until roughly 110 generations (and solutions are assumed to improve beyond that). Compared with the single glider case shown in Fig. 3, there is less clustering of the GA solutions, an indication that with multiple gliders, the solution is less likely to be trapped within a local minima. For this case, the best solution has a cost function that is substantially better than the other 19 simulations. The solution set (upper right panel of Fig. 5) reveals that there is significant spatial clustering of solutions in the local minima of the estimated cost function morphology. Several locations are sampled by gliders from every solution. Note that gliders that sample unique positions of the domain are generally associated with poorer solutions (blue).

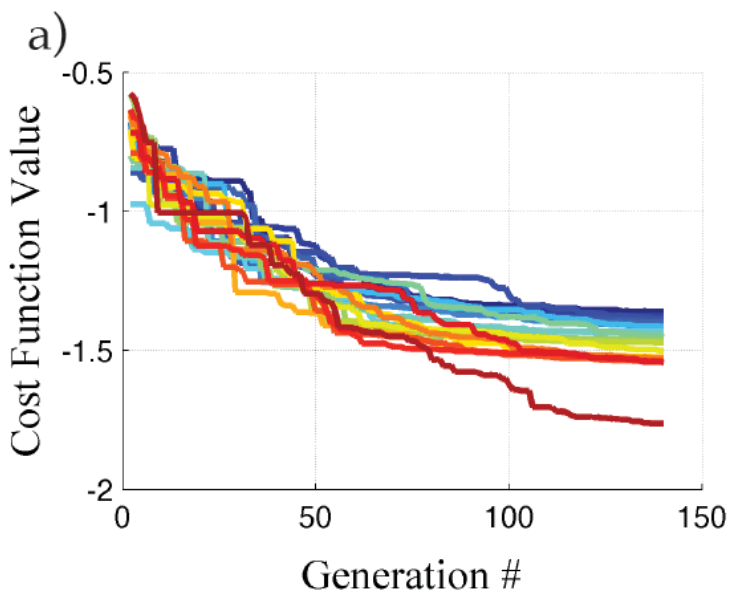

b)

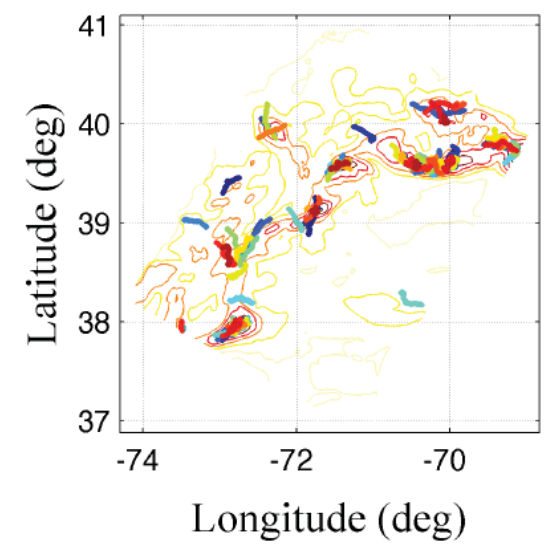

c)

d)
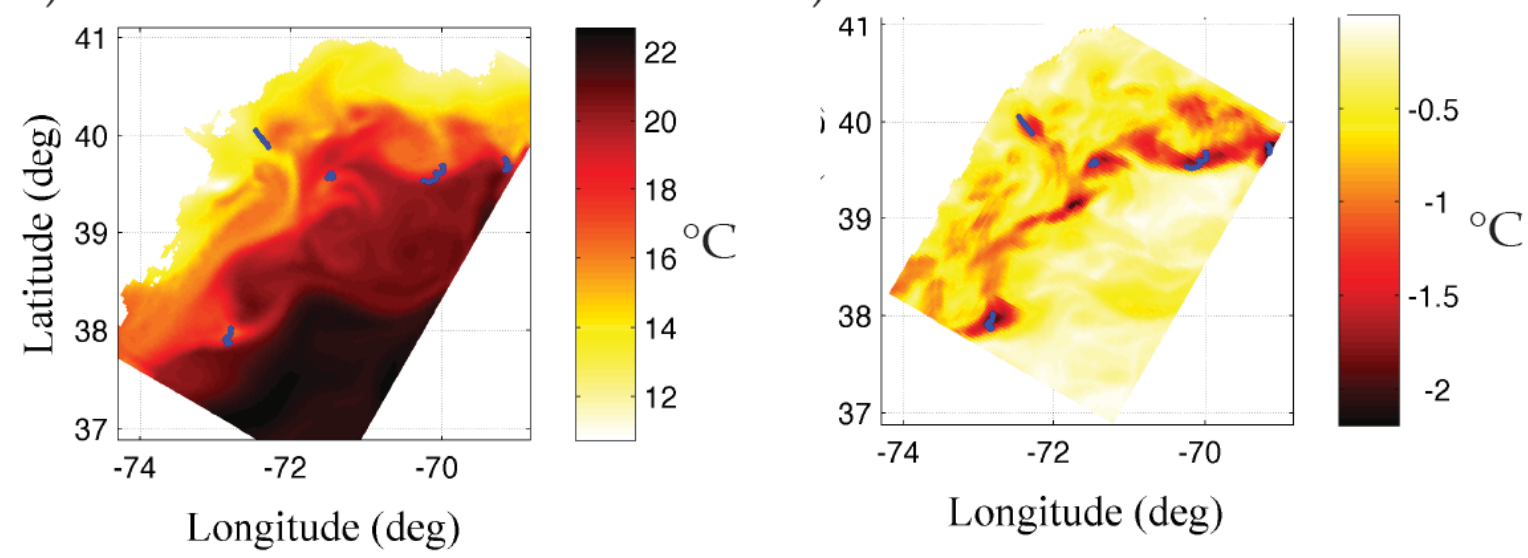

Figure 5. Aug 24-27 5-glider results for the $F_{\sigma}$ cost function. a) Cost function convergence vs. generation number. b) Solution set of locations of each GA solution. c) Best solution $(\mathrm{C}=-1.763)$ glider locations plotted on the temporally averaged $T_{\sigma}$ surface. d) Best solution plotted on the estimated cost function morphology.

The lower two panels of Fig. 5 plot the GA best solution over the temporally averaged $T_{\sigma}$ of the nearest-to mean ensemble and the estimated cost function morphology (left/right respectively). From the $T_{\sigma}$ plot we see that four gliders lie near the shelf-break with one inshore in the Hudson River region. From the cost function morphology plot we see that each glider passes through a local minimum of the cost function estimate. The five-glider solution best score (-1.763) is not as good as the best score found by a single glider rectangular brute force search (-2.1). This is expected because when a glider lies in the global minimum for the five-glider case, the other gliders must lie in other regions (with higher cost function contribution) due to the action of the distance-potential cost function. The effect of including currents can be seen in the track of the glider at longitude $70^{\circ} \mathrm{W}$ and $40^{\circ} \mathrm{N}$. This glider exhibits a double loop over 48 hours indicative of a tidal watch circle. 
The results for the Sep- $F_{f v}$ cost function are plotted in Fig. 6. As with Figure 5, there is a clustering of cost function values with generation number. The search converges by roughly 110 generations. The solution set, shown in the upper-right panel of Fig. 6 indicates that all solutions are oriented across local slopes and are clustered in four to six general regions. Unlike the model uncertainty case $F_{\sigma}$ shown above, in this case crossing the front is the important sampling feature, and each optimization yields glider tracks crossing the fronts that are present. For spatial variability cost functions the glider searches with large spatial extents (moving with the currents across fronts) are generally preferred.

The sample strategy of the best solution is plotted in the lower two panels (time averaged of the closest to mean $T_{\sigma}$ and estimated cost function morphology, left/right respectively). The algorithm has found solutions with all five gliders crossing fronts. It is evident from the estimated cost function morphology that each glider crosses a region where the estimated cost function morphology had a local minimum. The best solution score of -4.033 is significantly better than the brute force search solution of -2.5 , even with the score being the average for five gliders. This is due to the constraint that heading is fixed to 0 and 90 degrees in the brute force search. The GA can find glider orientations that cross the shelf-break front perpendicularly, thus raising the value of the standard deviation of the measured temperature and reducing the cost function score.

a)

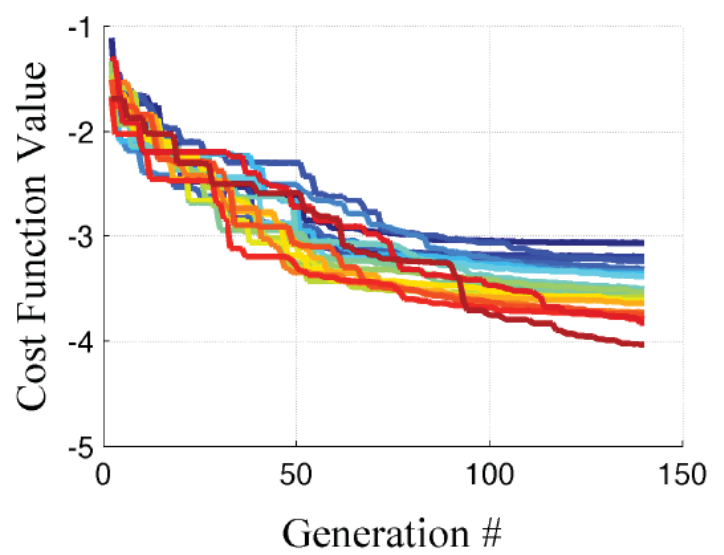

C)
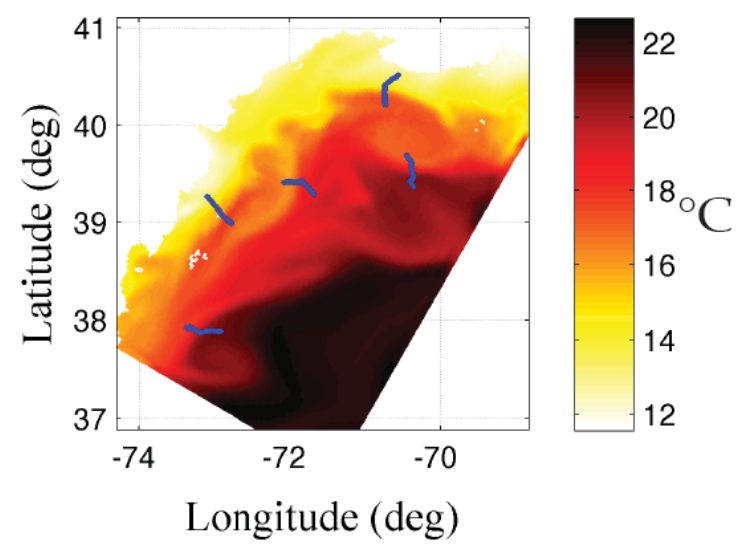

b)

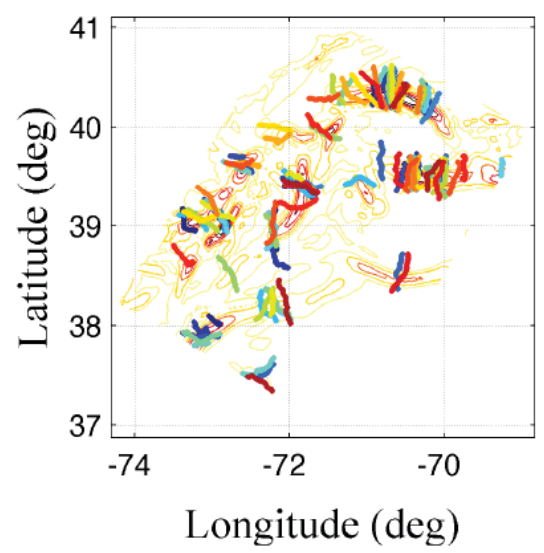

d)

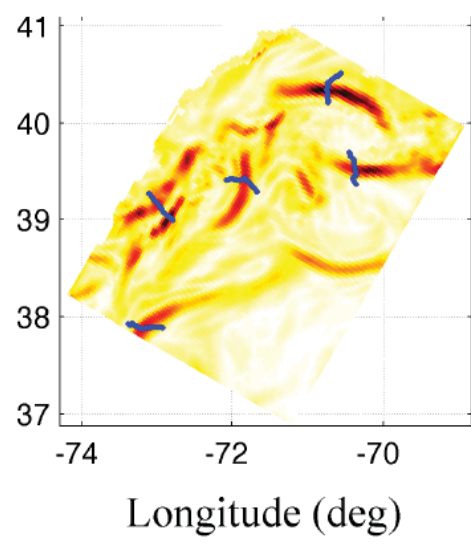

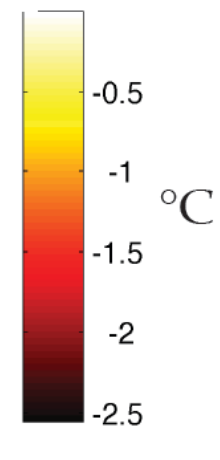

2.5

Figure 6. Sep 4-7 5-glider results for the $F_{f v}$ cost function. a) Cost function convergence vs. generation number. b) Solution set of locations of each GA solution. c) Best solution $(\mathrm{C}=-4.033)$ glider locations plotted on the temporally averaged $T_{\sigma}$ surface. d) Best solution plotted on the estimated cost function morphology.

In order to demonstrate the capabilities of the GA to find oceanographic regions of interest, in Fig. 7 the 'data' from the 'true ocean' along GA-selected sample tracks are plotted over the ensemble values. The upper row is for glider paths found using the GA with the model uncertainty $\left(F_{\sigma}\right)$ cost function and the lower is a set of tracks determined using the spatial variability $\left(F_{f i}\right)$ cost function. Note the significant ensemble spread from glider paths selected using the $F_{\sigma}$ cost function. This is as expected since the GA is optimizing for tracks that sample regions of high ensemble variability. For the lower row in Fig. 7, the spatial variability cost function $\left(F_{\sigma}\right)$ is used and the GA has selected tracks with large measurement variability (i.e. crossing fronts or traversing eddies). Most of the $F_{f v}$-optimized tracks lie along fronts, some with model ensemble variability (gliders 2 and 3 ), others without.

This raises the question of which sort of data to measure. Clearly from Fig. 7 the glider tracks selected using the $F_{\sigma}$ cost 
function can be used to select which of the ensembles is closer to the truth and assimilation of these values will reduce the ensemble spread (uncertainty). Some of these positions, however, have little temperature spread across the measured ensemble and are not associated with fronts. Paths found using the $F_{f v}$ cost function, by contrast, all cross major fronts yet some of these have little ensemble variability. Are measurements in locations where there is little dynamics but high-model uncertainty more valuable than measurements in regions that are highly dynamic, like across ocean fronts, yet there is little model uncertainty? This is an open question and an area of active research. The utility of the GA solution is that we can, for the moment, combine these cost functions.
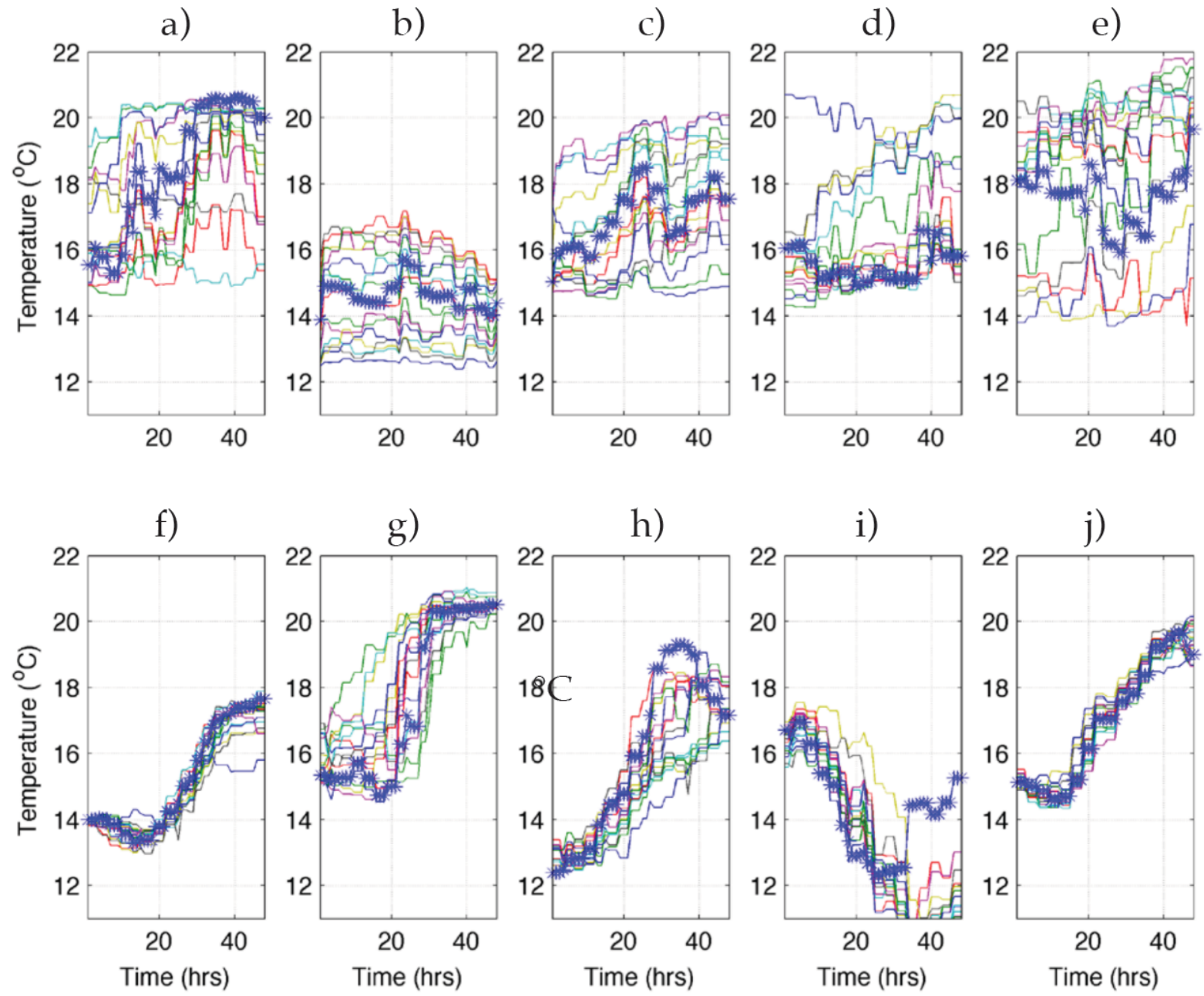

Figure 7. Aug 24-27 glider sample values of $T_{\sigma}$ for paths selected by model uncertainty $\left(F_{\sigma}\right)$ (upper row: a-e) and spatial variability $\left(F_{f v}\right)$ (lower row: f-j) from the ensembles (lines) and true ocean $(*)$. Clearly the $F_{\sigma}$ cost function is finding glider tracks that sample where the ensembles are most varied. The $F_{f v}$ cost function is finding solutions across dynamic regions, sometimes without much model variation.

\section{2) Global Cost Functions}

Here, five-glider sampling patterns are shown for optimizations involving global cost functions. For the Aug 24-27 and Sep 47 time periods, an ensemble of optimizations were performed using the normalized (uniformly) weighted sum of the $F_{\sigma}, F_{f v}, D$ and $B$ constituent cost functions. For the global cost function we expect the solution to contain glider sampling in regions with substantial inter-ensemble variability $\left(F_{\sigma}\right.$ cost function) as well as significant spatial variability compatible with features such as fronts (the $F_{f v}$ cost function). Fig. 8 shows results for Aug 24-27. The GA parameter set is identical to runs described in the previous section. Results converge by 110 generations. The collection of solutions reveals that cross-shelf tracks are favored, and that many of the tracks are clustered at the most offshore (southeast) front position where temperatures range from 16 to $18^{\circ} \mathrm{C}$. The tracks of the best solution gliders tend to cross fronts, but there is also a glider in the northeast corner, previously pointed out to be a location of significant inter-ensemble variability.

The numerical cost function result for the minimized (best) solution is -3.436 , lower than the brute force search score of -3.0 (read from the color scale of Fig 8.b). It is interesting to note that the global minimum encountered in the brute force (located in 
the center) is not selected by the GA best solution. It is likely that the average value of each of the glider solutions found is better than -3.0 so that placement of a glider track in this 'global minimum' would not improve the combined five-glider score.

a)

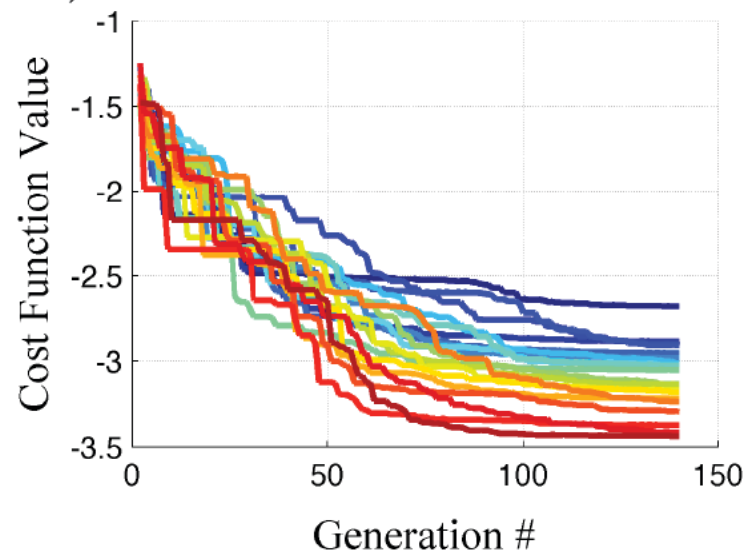

C)
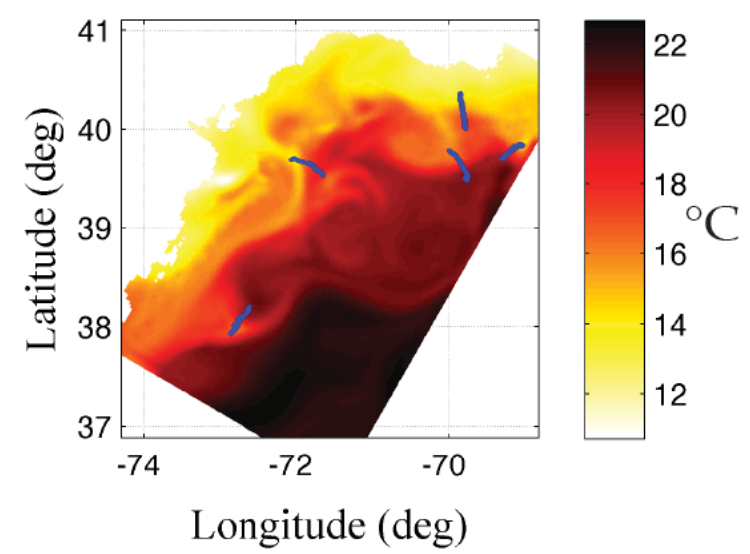

b)

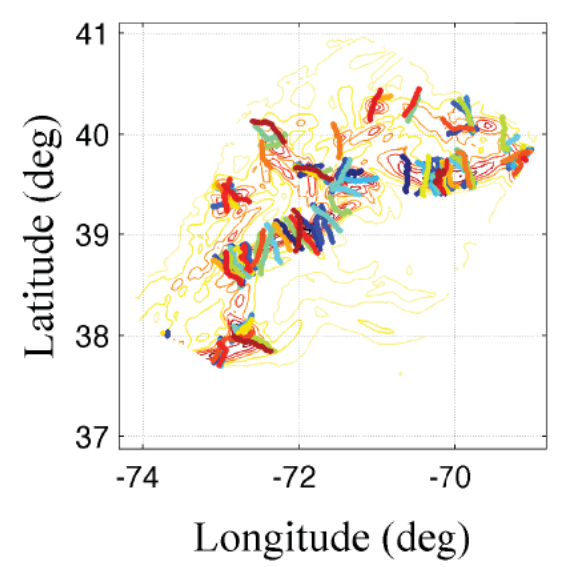

d)

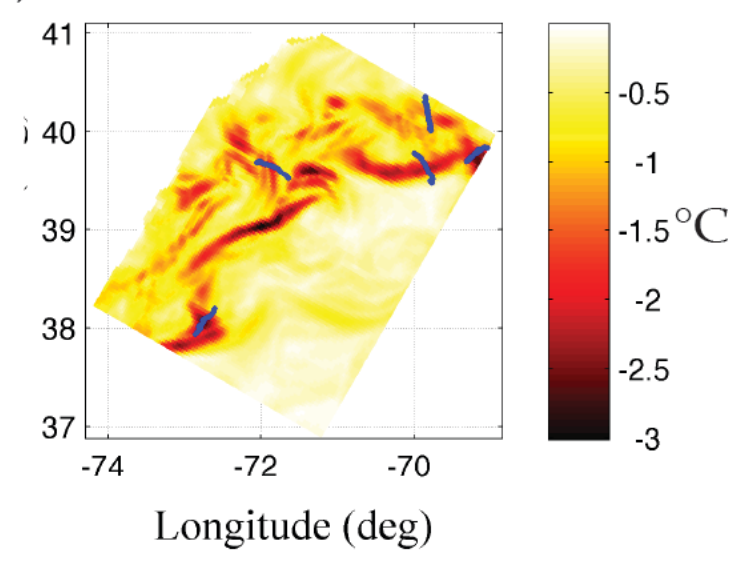

Figure 8. Aug 24-27 5-glider results for the global cost function. a) Cost function convergence vs. generation number. b) Solution set of locations of each GA solution. c) Best solution $(\mathrm{C}=-3.436)$ glider locations plotted on the temporally averaged $T_{\sigma}$ surface. d) Best solution plotted on the estimated cost function morphology.

The Sep 4-7 results using the global cost function are presented in Fig. 9. The convergence curves show little variability, settling on a rank ordering by generation 90 , but with continued improvement to 140 . The solution set reveals significant clustering of glider tracks across four or five main fronts. The best solution places three gliders (clearly separated by a correlation scale of $20 \mathrm{~km}$ ) crossing a front in the northern most area of the model domain. These three glider tracks could be reduced to two tracks or even a single track by increasing the correlation length to 50 or $100 \mathrm{~km}$. The GA best solution score of -3.642 is significantly better than the brute-force search solution of -2.6 (taken from the scale of Fig 9d). In fact, beyond 50 generations every GA solution has a better score than the brute-force search estimates, which have severely constrained track geometry. 
a)

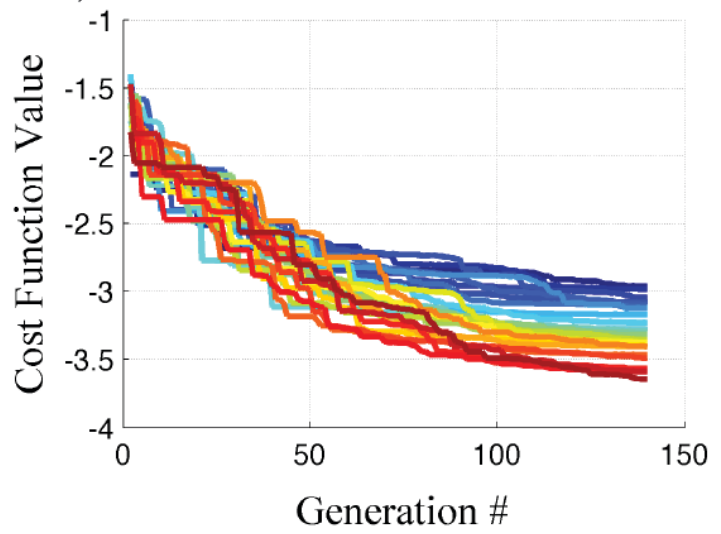

c)

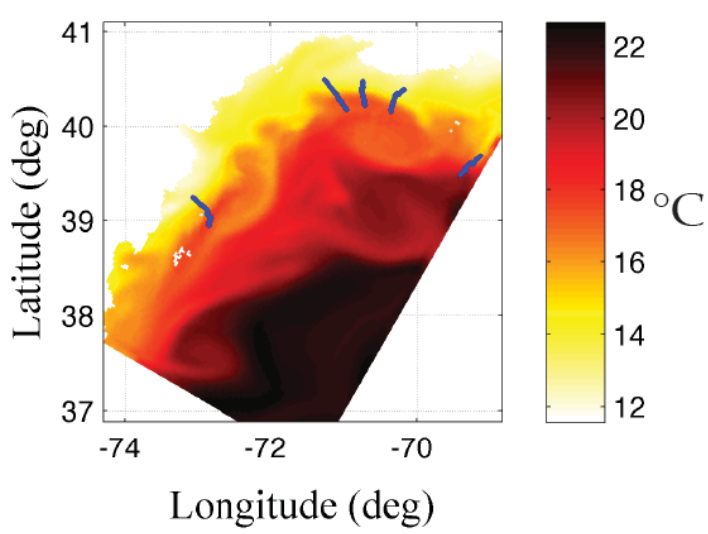

b)

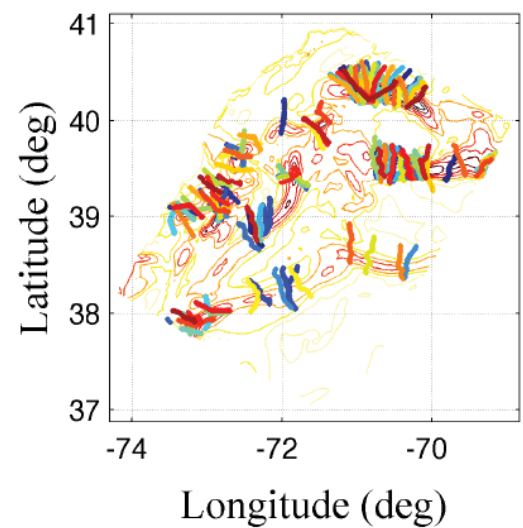

d)

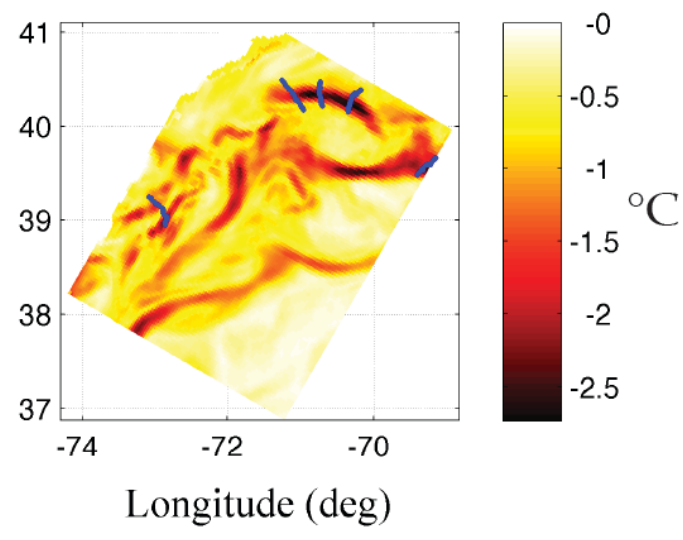

Figure 9. Sep 4-7 5-glider results for the global cost function. a) Cost function convergence vs. generation number. b) Solution set of locations of each GA solution. c) Best solution $(\mathrm{C}=-3.642)$ glider locations plotted on the temporally averaged $T_{\sigma}$ surface. d) Best solution plotted on the estimated cost function morphology.

\section{VALIDATION}

In Sections I-IV of this paper the method and behavior of the optimal sampling approach using the genetic algorithm has been presented. A goal of this paper is to validate that the platform laydown solutions determined by the GA are indeed optimal. Unlike the Mixed Linear Programming method (Yilmaz 2005; Yilmaz, et al. 2008), and simulated annealing (Collins and Kuperman 1991) mathematical proof of optimality for the GA is not possible. We settle for demonstration that the GA solutions provide value added and are better than other sampling approaches. Two separate approaches to measuring value added are applied. The first is the 'shooting method', where the ensemble that has predicted values closest to the 'measured' values is selected as the model forecast field and the second is the full assimilation of 'measured' data. The second approach is a classic Observational Systems Simulation Experiment (OSSE).

Two alternative sampling strategies will be compared with the GA-based search method results to evaluate adaptive sampling performance. One strategy, termed the random deployment, is a random selection from a viable set of searches generated using random initial conditions with a uniform pdf. The first generation of a particular GA solution is used. Recall the search is initiated from random set (uniform pdf) of each search parameter. From this set, the distance-potential $(D)$ and boundary $(B)$ cost functions are evaluated. Search strategies that survive this pass (meaning the gliders don't run aground and are not too close to each other in time or space) form a set from which the random search is drawn. This approach can be thought of as a coverage-constrained, optimal operating depth approach. It is the optimal strategy that was applied in the AOSN-II experiments in Monterrey Bay. The second strategy is a simplified grid approach based upon intuition of the region's physical oceanography. For shelf-break front environments, the gliders are spread out spatially and set to transect the region of dynamical oceanography. This grid approach is the current technique used in the absence of adaptive sampling. During the SW06 field test, six gliders were deployed by Rutgers University. Five of them made transects across the shelf-break front, perpendicular to the shelf. The gliders sampled from water depth $60 \mathrm{~m}$ to $600 \mathrm{~m}$ and then turned back. For the grid sampling approach, five gliders have been placed manually roughly equidistant from each other throughout the operational region, also ranging from $60 \mathrm{~m}$ to the $600 \mathrm{~m}$ 
deep locations. The distance between these isobaths can be significantly larger than $40 \mathrm{~km}$, so a random start position was selected. An ensemble of 20 grid sampling paths was created.

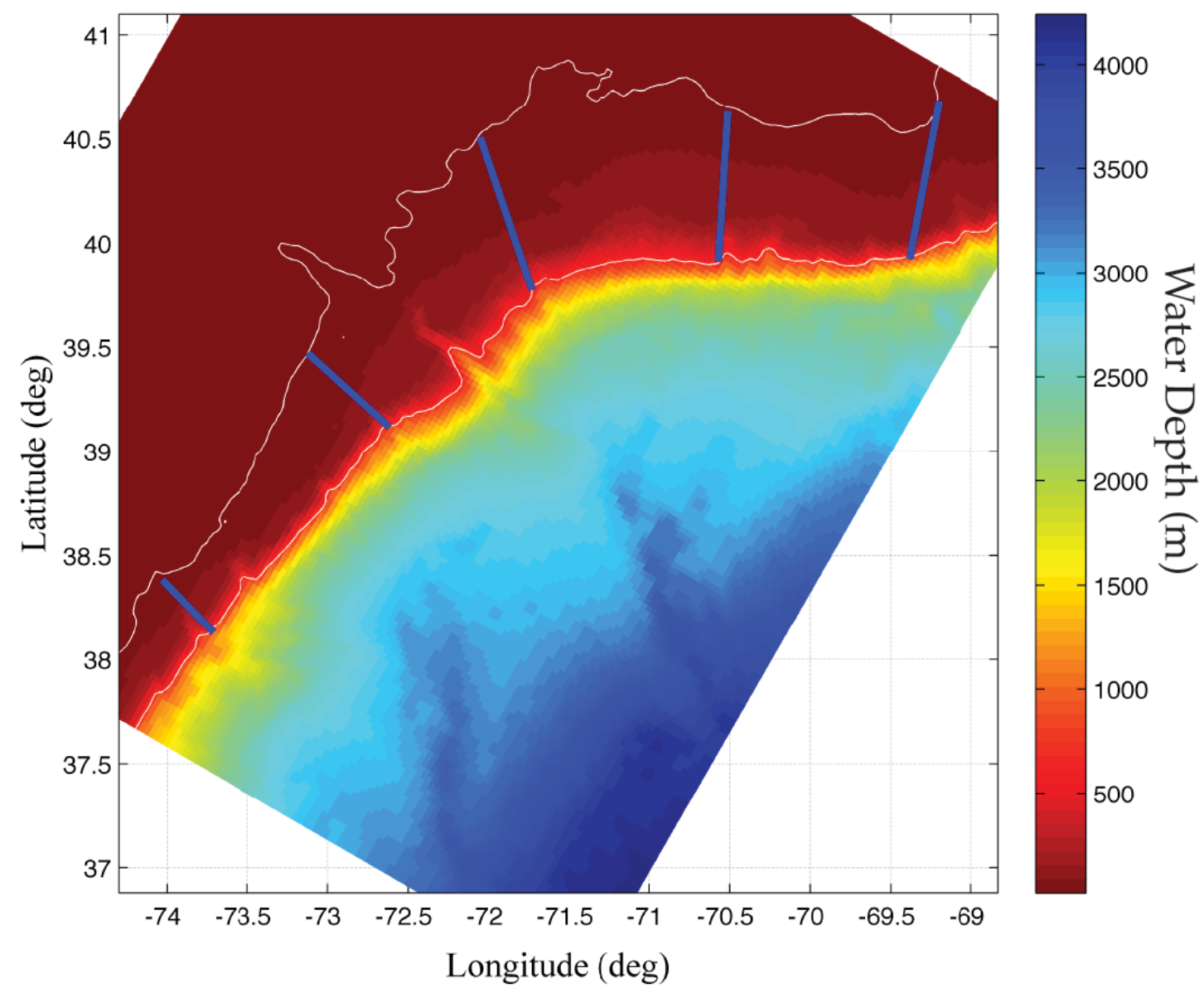

Figure 10. Paths selected for the "grid" sampling approach, based upon oceanographic intuition. Each path consists of a cross-slope sample from the 60m isobath to the $600 \mathrm{~m}$ isobath. A 48 -hour straight path sample was taken from each line (this is a sub-set of the blue lines shown).

\section{A. Shooting Method}

A means of evaluating various glider-sampling strategies is sought that does not require a full data-assimilation computation. A candidate approach, referred to as the "shooting method", is presented here. The method involves the selection of the 'best' candidate from an ensemble of ocean model forecasts using measurements from a 'true' ocean. The goal here is to compare three different techniques for selecting the measurement paths. For this demonstration, the ensemble and 'true' oceans described in Section II will be used. The true ocean has been generated by assimilating all available data from the SW06 experiment. For each forecast ensemble, there is no assimilated data. The 'best' ensemble is selected by comparing the forecast fields with the 'measurements' taken from the 'true' ocean. Measures of the correlation of the best ensemble with the 'true' ocean are used to evaluate different sampling strategies. This comparison is a harsh test compared to the full data assimilation approach because temporal and spatial coherence scales permit the assimilation of measurements to affect non-local fields.

The first step in the procedure is to use a search strategy $(i=\{G A$, random, grid $\})$ to determine a glider sampling strategy $x^{i}(t)$. Unlike the GA search strategy, the random and grid approaches have no a-priori method to differentiate between different candidate strategies. In order to resolve this conflict (and not choose a particularly good, or particularly bad search strategy) an ensemble of search strategies will be computed and their subsequent utility will be averaged. For each search strategy the measurement values $T_{\text {data }}{ }^{i}(t)$ are extracted from the "true" ocean. Forecasts for each search strategy are then generated for each ensemble $T_{\text {forecast }}{ }^{i}(t)$. The root-mean-square-error (RMSE) is computed between the forecast field and the measured data for each ensemble. The selected, or 'best', forecast for each search-strategy option is the ensemble with the minimum RMSE. The final step involves scoring the search-strategies, by computing the utility. The utility is the entire-domain RMSE computed for each search strategy. For the random and grid search strategies, the utility is averaged across each possible search strategy. 
The following method was used to select a search from the 100 random search strategies in the random set. First all searches which yielded ensemble 3 for the August 24-27 time period were selected. This included 31 search strategies. The GA cost function was evaluated for those searches with a cost function value of greater than 20 were discarded. These solutions either had gliders run ashore (the boundary cost function), or gliders lay on top of each other (the distance-potential cost function). A search strategy was chosen randomly from the remaining (26). The first search within the grid ensemble was chosen since, by definition, all of these paths satisfy the boundary and distance-potential constraints. The search paths used for data-assimilation are shown in Fig. 11 for the August (left panel) and September (right panel) time periods. The grid and random search paths are the same for both time periods. For illustration, the cost function values using the $G A$ cost function for each 5-glider search strategy were computed. For the August time period the GA, grid, and random cost function values (these are RMS Temp) are $3.44{ }^{\circ} \mathrm{C},-1.33{ }^{\circ} \mathrm{C}$ and $-0.46^{\circ} \mathrm{C}$, respectively. For the September time period the GA, grid, and random cost function values are $3.59^{\circ} \mathrm{C},-1.89^{\circ} \mathrm{C}$ and $-0.67{ }^{\circ} \mathrm{C}$, respectively. These results demonstrate how the GA has placed gliders at positions where there is substantially more ensemble variability and spatial variability.

a)

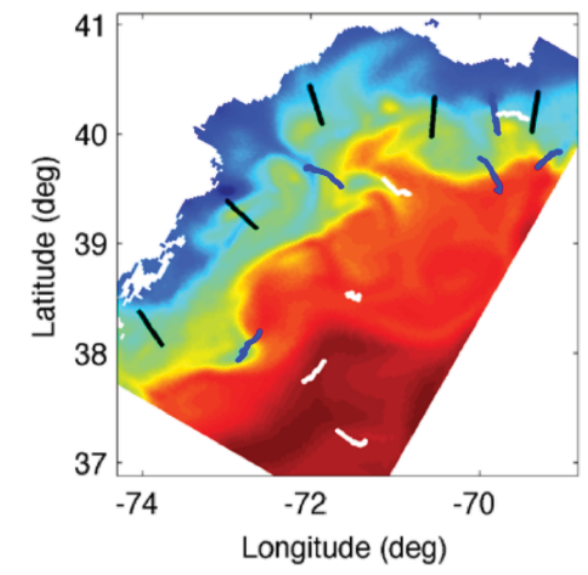

b)

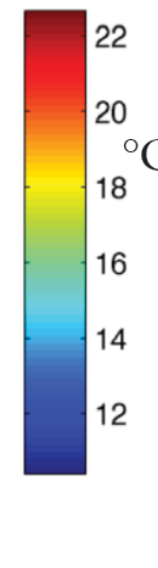

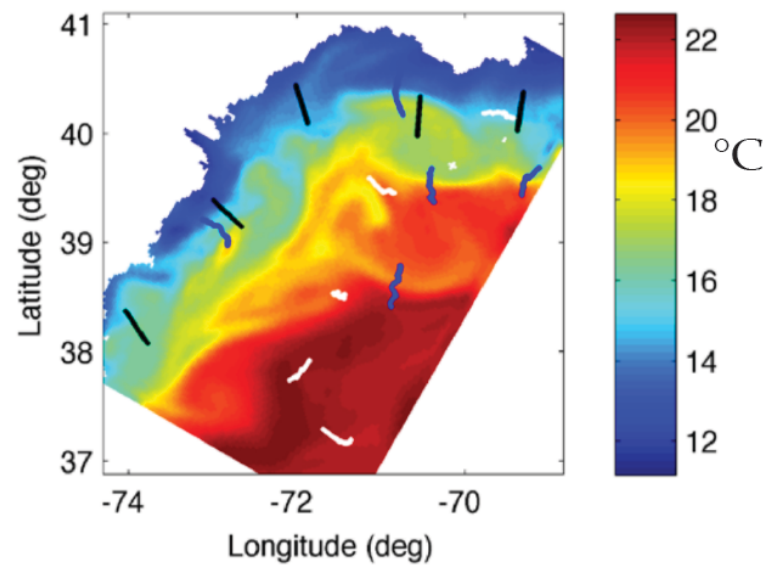

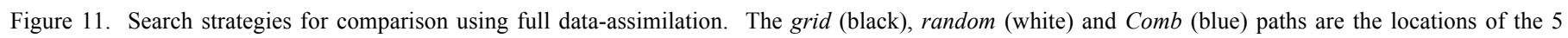
gliders over the 48 hours of data collection. The locations are overlaid on the $T_{\sigma}$ surface for August 24-27 (a) and September 4-7 (b).

For a so-called 'optimal' sampling strategy, the ensemble with minimum along the tracks should also have the domain-wide minimum. Thus, the tracks would provide representative sampling of the domain, sufficient to determine which ensemble member was closest to the truth. This is a method for using data to direct computational modeling results towards the most reliable attainable solution without the expensive step of assimilating data. To summarize, the procedure is as follows:

1. Optimization Algorithm: Generate sampling tracks (GA, grid, random) and take "measurements"

$$
\begin{aligned}
& \vec{x}^{G A} \Rightarrow \vec{T}_{\text {data }}^{\text {GA }} \\
& \vec{x}_{i}^{\text {grid }} \Longrightarrow \vec{T}_{\text {data }}^{\text {grid }} \\
& \vec{x}_{i}^{\text {rand }} \Rightarrow \vec{T}_{\text {data }}^{\text {rand }}
\end{aligned}
$$

2. Rank forecast ensembles by computing RMSE along tracks $C$

$$
\begin{aligned}
& C_{n}^{G A}=\sqrt{\left\langle\left(\vec{T}_{\text {data }}^{\text {GA }}-\tilde{T}_{\text {data }}^{\text {GA }}\right)^{2}\right\rangle} \\
& C_{n}^{\text {grid }}=\sqrt{\left\langle\left(\vec{T}_{\text {data }}^{\text {grid }}-\tilde{T}_{\text {data }}^{\text {grid }}\right)^{2}\right\rangle} \\
& C_{n}^{\text {rand }}=\sqrt{\left\langle\left(\vec{T}_{\text {data }}^{\text {rand }}-\tilde{T}_{\text {data }}^{\text {rand }}\right)^{2}\right\rangle}
\end{aligned}
$$

3. Select 'best' ensemble ocean with the minimum measured track RMSE 
4. Compute Utility (U) - domain wide RMSE for selected ensemble

$$
\begin{aligned}
& U^{G A}=\sqrt{\left\langle\left(\vec{T}_{\text {true }}-\tilde{T}_{n}^{G A}\right)^{2}\right\rangle} \\
& U_{i}^{\text {grid }}=\sqrt{\left\langle\left(\vec{T}_{n}^{\text {grid }}-\tilde{T}_{i, n}^{\text {grid }}\right)^{2}\right\rangle} \\
& U_{i}^{\text {rand }}=\sqrt{\left\langle\left(\vec{T}_{\text {true }}-\tilde{T}_{i, n}^{\text {rand }}\right)^{2}\right\rangle}
\end{aligned}
$$

5. For random and grid search strategies, average $U$, across various solutions $(i)$. Compare the $U$ various search strategies.

There are several options for handling the vertical averaging of the temperature fields in the computation of the RMSE. These include using the entire water column (all depths), the surface average $(\mathrm{z}<50 \mathrm{~m})$ and the $T_{\sigma}$ surface. The $T_{\sigma}$ surface is chosen because the GA cost functions were built from this surface. Physical oceanographic processes that drive the RMSE at depth or near the surface are not well sampled by this particular implementation of the GA and are therefore not suitable for comparison. An illustration of the results is shown in Fig. 12. For the August (left) and September (right) time periods, the RMSE values for the: model domain (blue solid line); the global GA solution, which matches both cost functions of equal weighting, (magenta line); the mean random sampling (cyan line); and, the GA best (thick*), are plotted. For the August time period, according to the model domain RMSE, ensemble member 3 is closest to the true state with a value of $0.71{ }^{\circ} \mathrm{C}$. Realizations 5 , 9-12 and 15 all have values near $0.79{ }^{\circ} \mathrm{C}$. The GA best and random sampling means select the best realization (number 3 ). The random search approach is almost perfectly correlated with the model-domain RMSE. The GA is less correlated, possibly because of sampling within dynamic regions (note the significantly higher data-forecast RMSE values) and possibly because the sample size of 20 is not large enough to ensure proper statistics. For the September time period (right panel), according to the model domain RMSE, realization 11 is the best with a value of 0.64 , while realizations $2-3,9-10,12-13$, and 15 have values near $0.70{ }^{\circ} \mathrm{C}$. The grid results are not shown for clarity. The mean grid RMSE values were poorly correlated with the model-domain RMSE. In particular for the Sep. time period, the grid method selected exclusively realizations 4 and 14, both with poor model-domain RMSE values $\left(\sim 0.75^{\circ} \mathrm{C}\right)$. This begins to highlight the difficulty in 'selecting' an ocean based upon five measurements over a 400 x $400 \mathrm{~km}$ region. 
a)

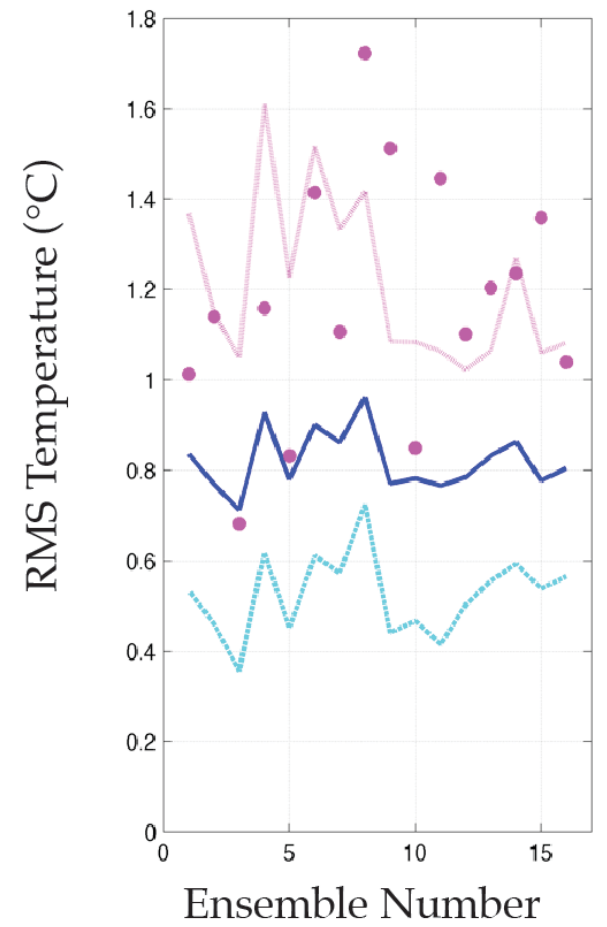

b)

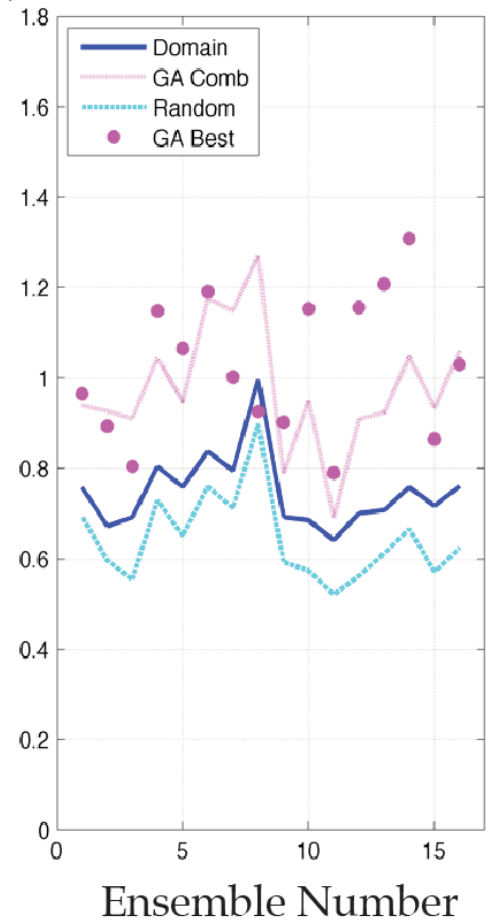

Figure 12. Comparison of RMSE for GA sampling measurements (best *; random - cyan line; grid - magenta line) and region wide model-true ocean (blue line). Panel a) is for the Aug 24-27 frame. Panel b) is for Sep 4-7. Note the spread in rank and the correlation between the GA measurements RMSE and the model domain computations.

The RMSE averages from the two time periods are presented in Table 1. The average of all the ensemble members is presented at the top. Without any sampling, a random selection of an ensemble to use as the model forecast would yield this value as the domain wide RMSE. Results for the best GA solution and the mean of all the GA solutions are presented, as well as for the mean of the grid and random sample strategy solutions.

TABLE 1. RMSE Averages $\left({ }^{\circ} \mathrm{C}\right)$

\begin{tabular}{|c|c|c|}
\hline Sampling & August 24-27 & September 4-7 \\
\hline$<\mid$ Ens-True $\mid>$ & 0.82063 & 0.74857 \\
\hline Global GA (best) & 0.71207 & 0.64251 \\
\hline$F_{\sigma}$ (best) & 0.77718 & 0.67231 \\
\hline$F_{f v}$ (best) & 0.77718 & 0.75846 \\
\hline$<$ grid $>$ & 0.77572 & 0.74957 \\
\hline$<$ random $>$ & 0.76348 & 0.70419 \\
\hline
\end{tabular}

The very poor performance of the grid sampling during the September time period can be explained by the fact that the tropical storm Ernesto drove much of the shelf-break front out into deeper water. All grid sample paths are now well inshore of the dynamic oceanography that is a valuable discriminator between the ensemble members. The general difference between the best and the mean GA solutions is an indication that each GA solution is selecting a different ocean state. This indicates that although the cost functions' final values are very close and the glider positions are located in qualitatively similar regions of the ocean (along the fronts), they produce different results. When we look at the error surface map in Fig. 13 we see that the spatial scales of these error values are quite small ( $\sim$ a Rossby radius). Therefore some gliders will select a large region of error while others will miss these regions, even though both are measuring the front. The result is that the selection of a particular ensemble 
is effectively random. This shows that we do not have enough gliders to uniquely sample the environment and differentiate accurately between various ocean states.

a)

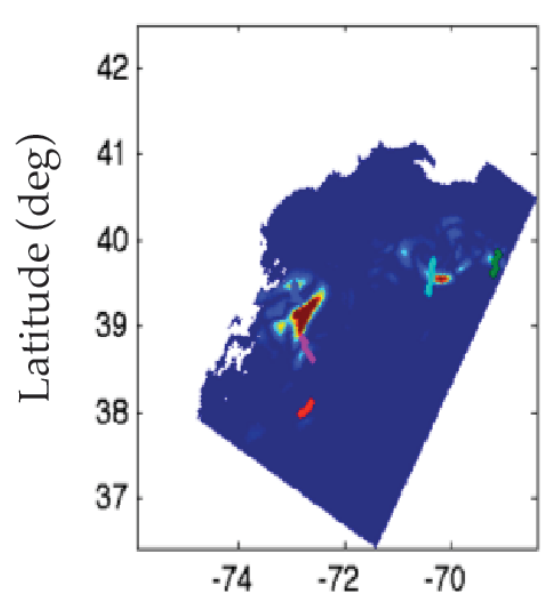

c)

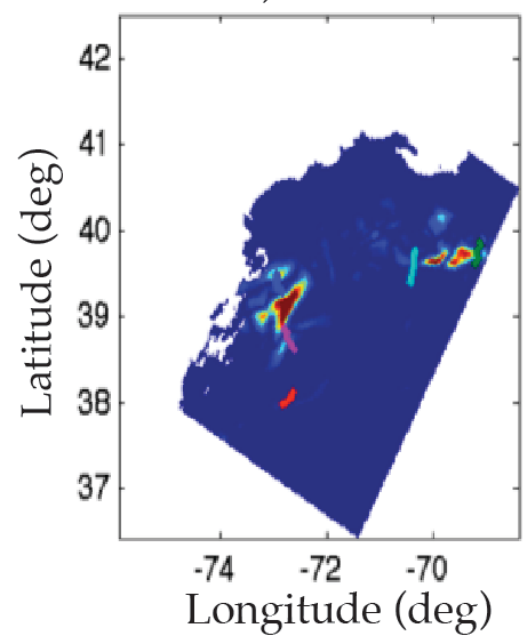

b)

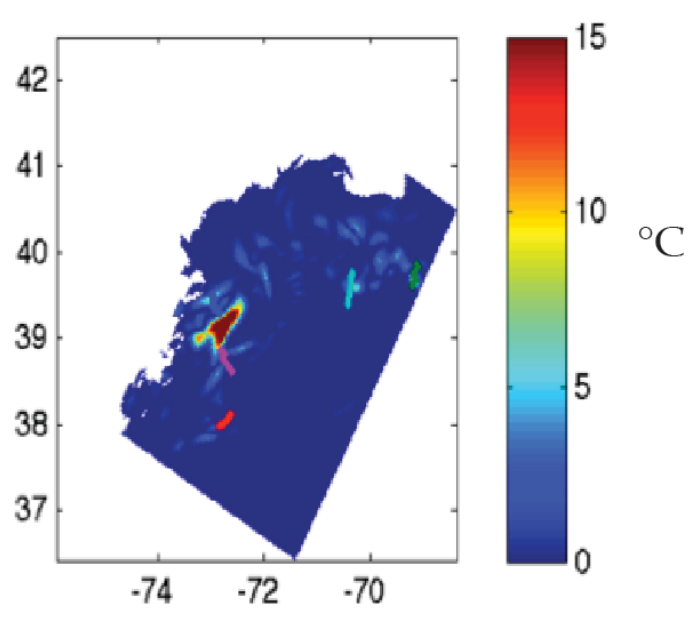

d)

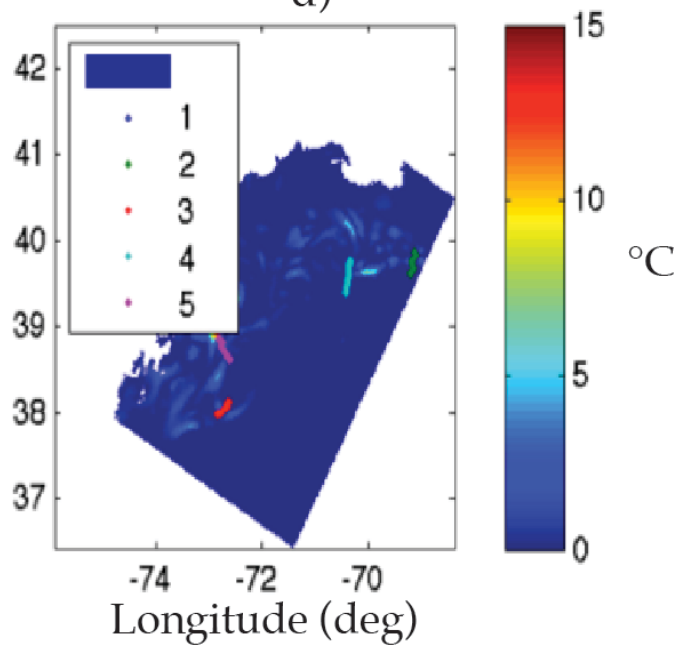

Figure 13. Error Maps for ensembles for August a) 3, b) 11, c) 12 and d) 16. The glider mean measurement RMSE are 1.46, 1.35, 1.31 and 0.99, respectively.

When looking at the error maps for August 24-27 (Fig. 13) it is clear that realization (ensemble member) 3 is the best and it has regions of error located near the shelf-break front in the Northeast. This is a region well sampled by the GA and if one of these hot-spots is sampled by a glider, this ensemble will be rejected. The random sampling however is much less likely to sample this region. The area of the hot-spot region divided by the area of the entire model domain is smaller than $1 / 10$. This means that the random sampling is less likely to reject ensemble member 3, or conversely, is more likely to reject all members besides 3. For the 100 random sampling runs 35 of them selected member 3 .

Note that the 'shooting' method is not a proper test of sampling strategies for data assimilation. The 'shooting' method approach selects the ocean ensembles that have minimum RMSE between the measurements and the models. Collecting data for assimilation into a model from these areas would yield data closest to the true ocean, which will have the smallest impact on the model.

For the shooting method experiment, data were taken from observations of the 'true' ocean along optimization-derived fiveglider sample paths, and for control purposes, along alternative five-glider sampling paths. The RMSE $\left(T_{\sigma}\right)$ between the observations and the model forecasts were computed and the minimum RMSE member of the forecast ensemble was selected as the "best" fit ocean. To compare the value added of various sampling networks the model domain wide RMSE $\left(T_{\sigma}\right)$ was computed for each ensemble. For the August 24-27 time period, the ensemble RMSE was 0.82. The GA-derived results for the Global cost function were 0.71 and 0.78 for the best and mean, respectively. The control sampling scheme derived from oceanographic intuition (perpendicular to the shelf break from $60-600 \mathrm{~m}$ ) and a random sampling scheme produced best values of 
0.78 and 0.76, respectively. For the September 4-7 time period, the ensemble RMSE was 0.75 . The GA-derived results for the Global cost function were 0.64 and 0.68 for the best and mean, respectively. Using a sampling scheme derived from oceanographic intuition and a random sampling produced best values of 0.75 and 0.70 , respectively. The best GA-derived sampling scheme selects the best ocean (smallest model domain RMSE) in all cases and is a significant positive result. The mean of the GA-derived results, however, performs comparably to the random sampling. With the exception of the Septembergridded sampling scheme, all of the sampling methods reduce the RMSE. The grid oceanographic sampling scheme suffers in the September time period because all of the dynamic oceanography shifts off the shelf-break as a result of the strong winds encountered during tropical storm Ernesto. A significant question is "Why does the random sampling scheme perform relatively well?". Both the grid pattern and the GA-based searches preferentially select sampling paths that are in the shelfbreak, a region of high oceanographic variability. The regions of high RMSE, even for the best ocean, are in this area. If a particular sample happens to land on one of these hot spots, the 'shooting method' will penalize this realization for the local RMSE, even though it has the minimum domain RMSE. The random sampling scheme is less likely to fall on a hot spot and reject the best oceans. This selection process is very local (there is no assimilation and therefore no advection) and is very sensitive to the exact positions of the gliders.

\section{B. Observational Systems Simulation Experiment}

In order to evaluate the value added from sampling and then assimilating the data (the truly optimal adaptive sampling problem), we proceed to assimilate data from the 'true' ocean using samples selected from the GA, random, and grid search algorithms.

The OSSE was completed by sampling 6 data sets from the simulated true ocean, using the 3 sampling methods in both the pre- Ernesto (24-27 Aug, 2006) and post-Ernesto (4-7 Sep, 2006) time periods. Each of these data sets was then assimilated into each of the 17 ensemble members over the first 2 days of the simulations (102 simulations in total). On the third simulation day, root mean square (RMS) errors and pattern correlation coefficients (PCC) were calculated for temperature and salinity at three depths (0m-mixed layer; 30m-thermocline; 100m-deep) to evaluate the impacts of assimilating the different sampling schemes. Figure 14 shows the RMS errors for the Genetic Algorithm and random sampling schemes relative to the RMS errors for the regular grid-sampling scheme. Also displayed in each panel is the average value for each curve. On average, the RMS temperature error for the Genetic Algorithm sampling is 6\% better than the regular grid in the August period (Fig. 14a), 1\% better in September (Fig. 14b). The PCC show similar results with 4\% improvement in August (Fig. 15a) and 2\% improvement in September (Fig. 15b). For Salinity the RMS improvements were 8\% in August (Fig. 16a), 5\% in September (Fig. 16b) and the PCC improvements were 6\% in August (Fig. 17a), 4\% in September (Fig. 17b).

These numbers may seem small, but they are in fact significant for three reasons: i) the amount of data simulated is very small (only a few gliders over 2 days in a large 500km-long domain); ii) the cost function for the GA does not focus per se on ocean predictive skill, but it still leads to improvements; and finally iii), obtaining comparable skill improvements solely from upgrading the model would require several man-years of effort, as quantified for example for atmospheric prediction systems. Hence, in this shelfbreak region, just a little bit of data well sampled using the GA instead of a regular grid sampling algorithm can lead to as significant improvements as updating the modeling system over several years.

a)
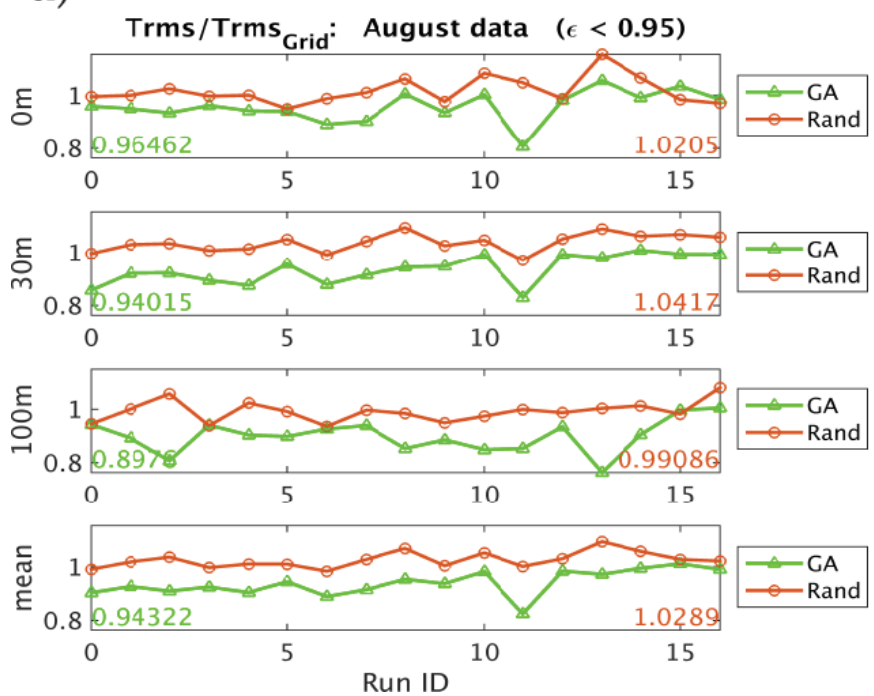

b)
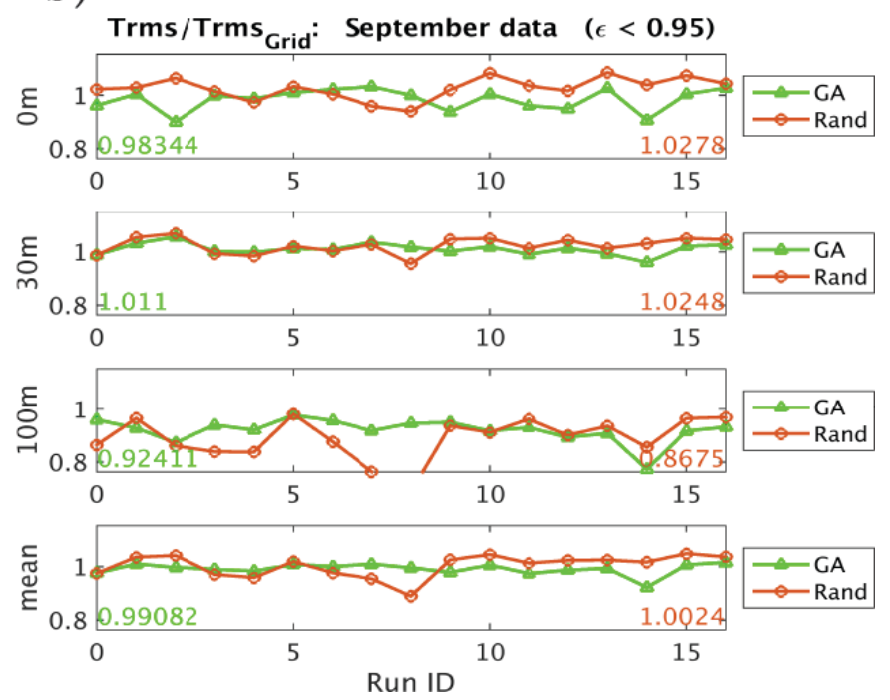

Figure 14. Temperature RMS errors for runs assimilating pseudo-data sampled according to the Genetic Algorithm and random starting points, relative to the temperature RMS errors for runs assimilating pseudo-data sampled on a regular grid. (a) Prior to tropical storm Ernesto, (b) after Ernesto. Each panel shows the 
relative RMS errors for each ensemble member. Also shown in each panel is the numerical value for the average of each curve.

a)
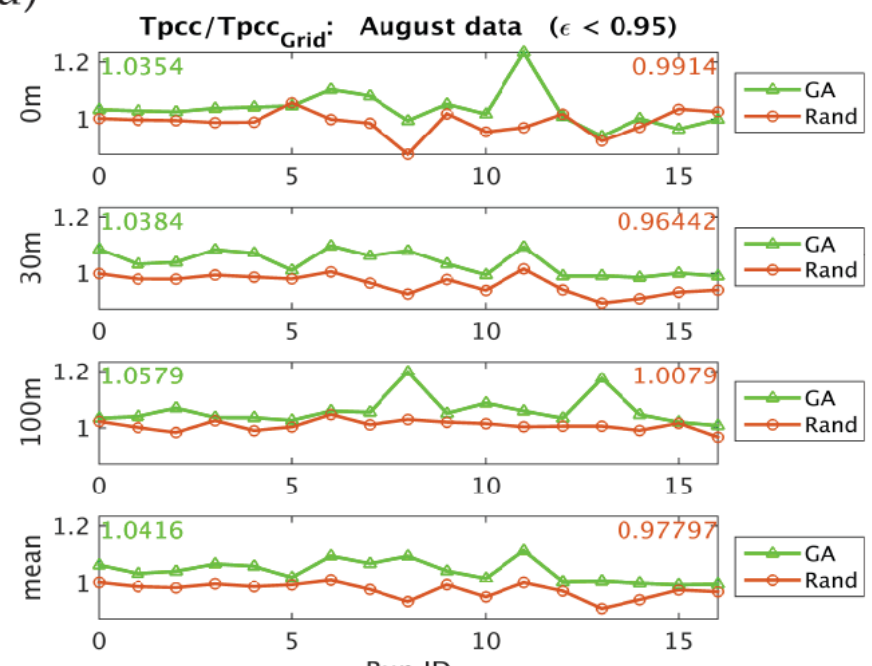

b)
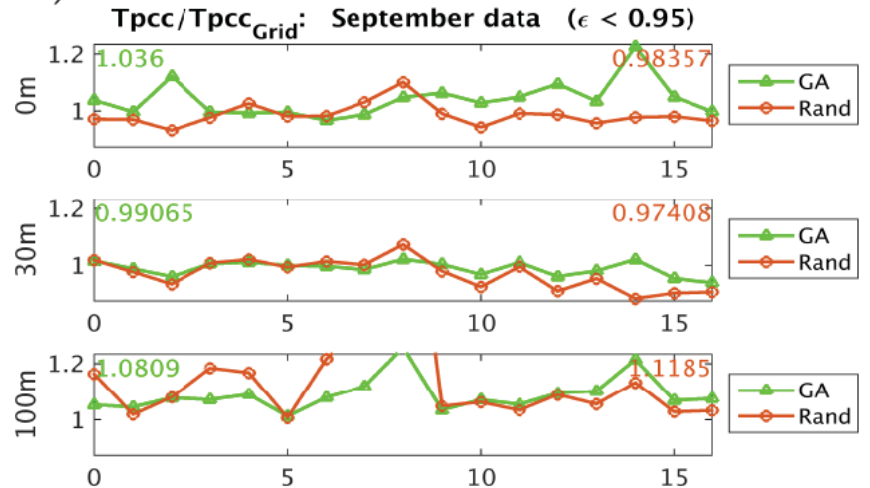

Figure 15. Temperature PCC for runs assimilating pseudo-data sampled according to the Genetic Algorithm and random starting points, relative to the temperature PCC for runs assimilating pseudo-data sampled on a regular grid. (a) Prior to tropical storm Ernesto, (b) after Ernesto. Each panel shows the relative PCC for each ensemble member. Also shown in each panel is the numerical value for the average of each curve.

a)
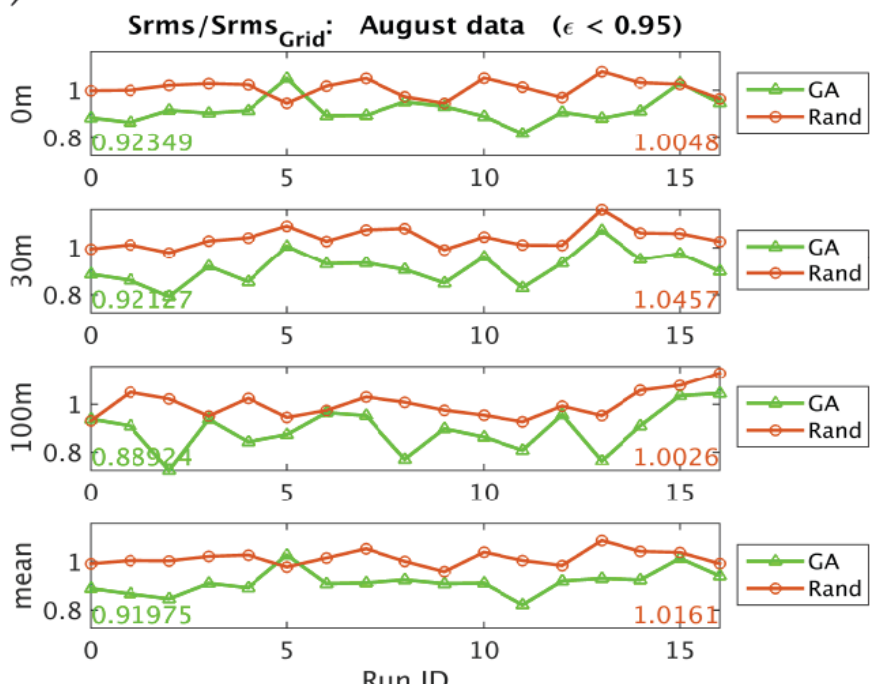

b)
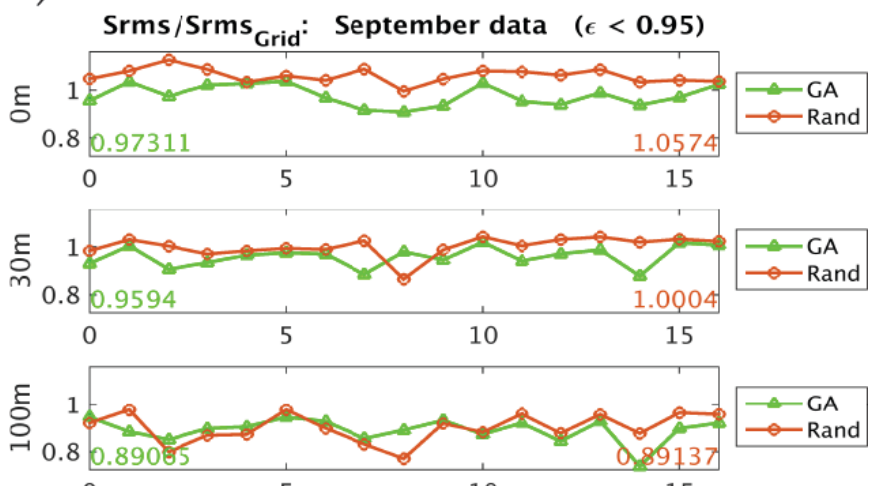

Figure 16. Salinity RMS errors for runs assimilating pseudo-data sampled according to the Genetic Algorithm and random starting points, relative to the salinity RMS errors for runs assimilating pseudo-data sampled on a regular grid. (a) Prior to tropical storm Ernesto, (b) after Ernesto. Each panel shows the relative RMS errors for each ensemble member. Also shown in each panel is the numerical value for the average of each curve. 
a)
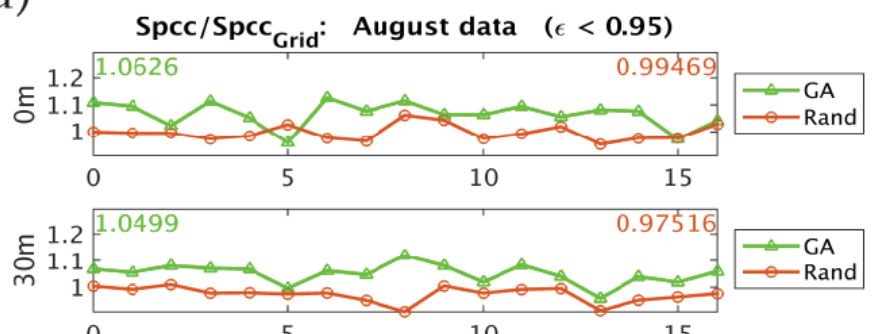

동 1.2
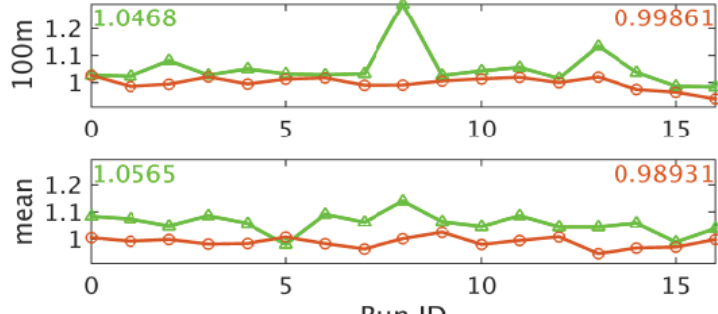

b)
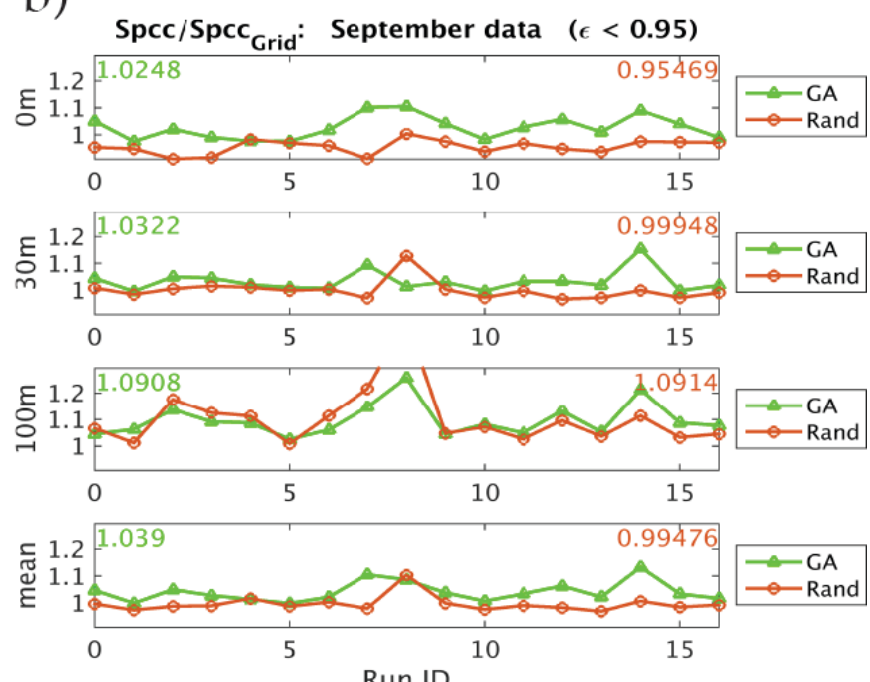

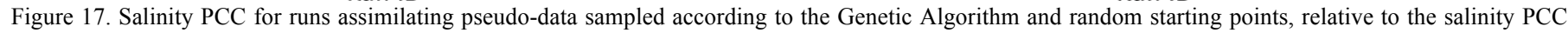

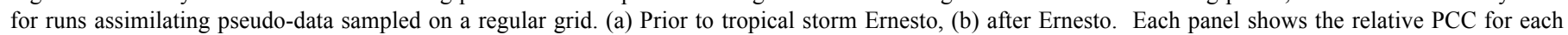
ensemble member. Also shown in each panel is the numerical value for the average of each curve.

Overall, the performance in salinity is a bit better than that for temperature, which is encouraging since the main density-driver of the flow in the region is salinity. Also, the increase in performance using the GA instead of a regular grid sampling algorithm is a bit larger before the storm than after the storm. This is in part because the density isopycnal along which most of the GA cost function terms are evaluated is better suited for the field before than after the storm. Ideally, the density isopycnal should be modified or several density isopycnals should be included in the cost functions so as to cover the range of dynamicallysignificant densities in the region.

\section{SUMMARY AND CONCLUSION}

In this paper a method for determining optimal paths for ocean sampling was presented. The term "optimal" refers to the paths that minimize a user-defined cost function within the specified time constraints. The problem is posed as a constrained non-linear optimization problem and is solved using an implementation of the Genetic Algorithm. The environment considered in this paper was the New Jersey area of the Mid-Atlantic Bight, where an experiment with extensive oceanographic sampling was conducted in the fall of 2006 (Shallow Water 06). Two time periods were used, one just prior (August 24-27) and one just after (September 4-7) the passage of tropical storm Ernesto. Using the Multidisciplinary Simulation, Estimation and Assimilation System (MSEAS), an ESSE ensemble of 17 model forecasts for each 3-day time period was created. These forecasts serve as the ocean field and uncertainty prediction prior to the collection of data. A "true" ocean model was computed by assimilating all of the measured oceanographic data into the set of model forecasts. The cost functions used in this paper included the ensemble variability $\left(F_{f v}\right)$, the spatial variability $\left(F_{\sigma}\right)$, the sum of the two $($ Global), a distance potential to prevent gliders from sampling the same body of water and a boundary function to prevent platforms from being deployed in less than 50 $\mathrm{m}$ of water.

The behavior of the GA technique was examined by using a single glider and single constituent cost functions. Searches using the GA were found to converge within 40 generations for a single glider and always found a minimum of the cost function, but sometimes this was only a local minimum. The results of GA searches were consistent with the estimates made of the cost function morphology. In cases with a strong global minimum $\left(F_{\sigma}\right)$, the method placed all of the gliders within this minimum. For morphologies with many local minima, near the value of the global minimum, the method did not always converge on the global minimum. This is likely a result of not fine-tuning the parameters of the search (number of generations, number of individuals, mutation rate, etc.) correctly. It should be pointed out that GA-based optimization, unlike simulated annealing, is not mathematically guaranteed to converge. The results presented here are consistent with previous results using the GA to perform non-linear optimization.

The five-glider GA runs demonstrated robustness to the local-minima problem addressed in the previous paragraph. When searching for optimal sampling geometries for a fleet of five gliders, the GA method was able to regularly place a glider in the global minimum, and place the other four gliders in local minima. The convergence was significantly slower ( 110 generations) because of the lack of settling into local minima. Cost function values for the five-glider GA generally converged but the spatial sampling was often spread out over regions of spatial and ensemble variability. In particular, several GA-based solutions with 
the same cost function values would place gliders along the same front, but at different locations. This is an example of equifinality, where different solutions yield qualitatively similar results.

Two tests were used to quantitatively compare the GA solution with a random sample solution and a control sampling scheme derived from oceanographic intuition (perpendicular to the shelf break from $60-600 \mathrm{~m}$ ). These tests were conducted for both time periods. A 'shooting method' evaluated the ensemble member chosen from observations taken using each sampling strategy and compared the root-mean-square-error (RMSE) between the true ocean and the selected ensemble member. The Genetic Algorithm was able to identify hot-spots of the model forecast and take selective measurements there, permitting adaptation to the movement of the dynamic oceanographic regions prior to and after tropical storm Ernesto. The improvement of the ocean forecast using the GA from this experiment was on the order of 18\%. A full OSSE was also conducted, with assimilation of measurements taken using the sampling strategies above. The GA was then found to yield a $4-8 \%$ improvement in the RMSE and pattern correlation coefficient (PCC) of the forecasts for Temperature and Salinity.

By using a combination of model forecast uncertainty and variability as the physics drivers of the selection of sampling regions, the adaptive sampling approach presented in this paper leads to a collection of data in regions which have an optimized impact on forecast accuracy.

ACKNOWLEDGMENTS. We are very thankful to W.G. Leslie for his help with the merging of atmospheric forcing fields used in the ocean simulations, to G. Gawarkiewicz and P. Abbot for their AWACS-SW06 ocean data, and to M. Taylor and J. Hare for their NMFS survey data. We also thank J. Evans, S. Glenn, and J. Wilkin for their real-time WRF atmospheric fluxes and the FNMOC teams for their own products. This work was supported in part by a Space and Naval Warfare Center (SPAWAR) SBIR program. PFJL and PJH are also grateful to the Office of Naval Research for partial support under grants N00014-14-1-0476 (Science of Autonomy LEARNS), N00014-11-1-0701 (MURI-IODA) and N00014-12-1-0944 (ONR6.2) to the Massachusetts Institute of Technology. TFD's contribution was funded by the SBIR and grant N00014- 11-1-0701 (MURI-IODA).

\section{REFERENCES}

Alvarez A. and B. Mourre, "Optimum sampling designs for a glider-mooring observing network," J. Atmos. Oceanic Technol., vol. 29, pp. 601-612, 2012

Berliner, L. M., Z.-Q. Lu and C. Snyder (1999). "Statistical Design for Adaptive Weather Observations." Journal of the Atmospheric Sciences 56(15): 2536-2552.

Bishop, C. H., B. J. Etherton and S. J. Majumdar (2001). "Adaptive Sampling with the Ensemble Transform Kalman Filter. Part I: Theoretical Aspects." Monthly Weather Review 129: 420-436.

Carrassi, A., A. Trevisan and F. Uboldi (2007). "Adaptive observations and assimilation in the unstable subspace by breeding on the dataassimilation system." Tellus A 59(1): 101-113.

Chapman, N. R. and J. F. Lynch (2010). "Special Issue on the 2006 Shallow Water Experiment." IEEE Journal of Oceanic Engineering 35(1): $1-2$.

Cococcioni, M., M. Lazzerini and P. F. J. Lermusiaux (2015). Adaptive Sampling Using Fleets of Underwater Gliders in the Presence of Fixed Buoys using a Constrained Clustering Algorithm. OCEANS 2015 MTS/IEEE. Washington DC, IEEE.

Collins, M. D. and W. A. Kuperman (1991). "Focalization: Environmental focusing and source localization." Journal of the Acoustical Society of America 90(3): 1410-1422.

Collette, Y. and Siarry, P., 2013. Multiobjective optimization: principles and case studies. Springer Science \& Business Media.

Curtin, T. B., J. G. Bellingham, J. Catipovic and D. Webb (1993). "Autonomous oceanographic sampling networks." Oceanography 6(3): 8694.

Edwards, C. A., A. M. Moore, I. Hoteit and B. D. Cornuelle (2015). "Regional ocean data assimilation." Annual review of marine science 7: 21-42.

Egbert, G. D. and S. Y. Erofeeva (2002). "Efficient Inverse Modeling of Barotropic Ocean Ties." Journal of Atmospheric and Oceanic Technology 19(2): 183-204.

Ferri G, Cococcioni M, A Alvarez "Mission Planning and Decision Support for Underwater Glider Networks: A Sampling on-Demand Approach," Sensors 16 (1), pp.28, 2015

Frolov, S., B. Garau and J. Bellingham (2014). "Can we do better than the grid survey: optimal synoptic surveys in presence of variable uncertainty and decorrelation scales." Journal of Geophysical Research: Oceans.

Gangopadhyay, A., A. R. Robinson, P. J. H. Haley, W. G. Leslie, C. J. Lozano, J. J. Bisagni and Z. Yu (2003). "Feature-oriented regional modeling and simulations in the Gulf of Maine and Georged Bank." Continental Shelf Research 23(3-4): 317-353.

Gerstoft, P. (1994). "Inversion of siesmoacoustic data using genetic algorithms and a posteriori probability distributions." Journal of the Acoustical Society of America 95(2): 770-782.

Gerstoft, P. and D. F. Gingras (1996). "Parameter estimation using multifrequency range-dependent acoustic data in shallow water." Journal of the Acoustical Society of America 99(5): 2839-2850.

Gerstoft, P., W. S. Hodgkiss, W. A. Kuperman and H. C. Song (2003). "Phenomenological and Global Optimization Inversion." IEEE Journal of Ocean Engineering 28(3): 342-354.

Goldberg, D. E. (1989). Genetic Algorithms in Search, Optimization and Machine Learning. Boston, Addison-Wesley.

Haley, P.J. Jr., P.F.J. Lermusiaux, A.R. Robinson, W.G. Leslie, O. Logutov, G. Cossarini, X.S. Liang, P. Moreno, S.R. Ramp, J.D. Doyle, J. Bellingham, F. Chavez, S. Johnston, 2009. Forecasting and Reanalysis in the Monterey Bay/California Current Region for the Autonomous 
Ocean Sampling Network-II Experiment. Special issue on AOSN-II, Deep Sea Research, Part II. ISSN 0967-0645, doi: 10.1016/j.dsr2.2008.08.010

Haley, P. J. H. and P. F. J. Lermusiaux (2010). "Multiscale two-way embedding schemes for free-surface primitive-equations in the Multidisciplinary Simulation, Estimation and Assimilation System." Ocean Dynamics 60: 1497-1537.

Haley, P.J., Jr., A. Agarwal, P.F.J. Lermusiaux, 2015. Optimizing Velocities and Transports for Complex Coastal Regions and Archipelagos. Ocean Modeling, 89, 1-28. doi:10.1016/j.ocemod.2015.02.005

Hamill, T. M., F. Yang, C. Cardinali and S. J. Majumdar (2013). "Impact of Targeted Winter Storm Reconnaissance Dropwindsonde Data on Midlatitude Numerical Weather Predictions." Monthly Weather Review 141: 2058-2065.

Heaney, K. D. and T. F. Duda (2006). Non-linear Optimization of Multi-Vehicle Ocean Sampling Networks for Cost-effective Ocean Prediction Systems. OCEANS'06 Asia Pacific, Singapore, IEEE.

Heaney, K. D., G. Gawarkiewicz, T. F. Duda and P. F. J. Lermusiaux (2007). "Non-linear Optimization of Autonomous Undersea Vehicle Sampling Strategies for Oceanographic Data-Assimilation." Journal of Field Robotics Special Issue on "Underwater Vehicles".

Hoteit, I., T. Hoar, G. Gopalakrishnan, N. Collins, J. Anderson, B. D. Cornuelle, A. Köhl and P. Heimbach (2013). "A MITgcm/DART ensemble analysis and prediction system with application to the Gulf of Mexico." Dynamics of Atmospheres and Oceans 63: 1-23.

Leonard, N. E., D. A. Paley, F. Lekien, R. Sepulchre, D. M. Fratantoni and R. E. Davis (2007). "Collective Motion, Sensor Networks, and Ocean Sampling." Proceedings of the IEEE 95(1): 48-74.

Lermusiaux, P. F. J. and A. R. Robinson (1999). "Data Assimilation via Error Subspace Statistical Estimation.Part I: Theory and Schemes." Monthly Weather Review 127(7): 1385-1407.

Lermusiaux, P. F. J. (2004). Application of the Error Subspace Statistical Estimation (ESSE) system to real-time error forecasting, data assimilation and adaptive sampling off the Central California Coast during AOSN-II. 20th Conference on Weather Analysis and Forecasting/16th Conference on Numerical Weather Prediction, Seattle.

Lermusiaux, P. F. J. (2006). Uncertainty Estimation and Prediction for the Interdisciplinary Ocean. Special Issue of the Journal of Computational Physics on "Uncertainty Quantification". J. Glimm and G. Karniadakis: 176-199.

Lermusiaux, P. F. J., C.-S. Chiu, G. G. Gawarkiewicz, P. Abbot, A. R. Robinson, R. N. Miller, P. J. Haley, W. G. Leslie, S. J. Majumdar, A. Pang and F. Lekien (2006a). Quantifying Uncertainties in Ocean Predictions. Oceanography, Special Issue on "Advances in Computational Oceanography". T. Paluskiewicz and S. Harper. 19: 92-105.

Lermusiaux, P.F.J., P. Malanotte-Rizzoli, D. Stammer, J. Carton, J. Cummings and A.M. Moore, 2006b. "Progress and Prospects of U.S. Data Assimilation in Ocean Research". Oceanography, Special issue on "Advances in Computational Oceanography", T. Paluszkiewicz and S. Harper, Eds., 19, 1, 172-183

Lermusiaux, P.F.J, 2007. Adaptive Modeling, Adaptive Data Assimilation and Adaptive Sampling. Refereed invited manuscript. Special issue on "Mathematical Issues and Challenges in Data Assimilation for Geophysical Systems: Interdisciplinary Perspectives". C.K.R.T. Jones and K. Ide, Eds. Physica D, Vol 230, 172-196, doi: 10.1016/j.physd.2007.02.014

Lermusiaux, P.F.J., P.J. Haley Jr. and N.K. Yilmaz, 2007. Environmental Prediction, Path Planning and Adaptive Sampling: Sensing and Modeling for Efficient Ocean Monitoring, Management and Pollution Control. Sea Technology, 48(9), 35-38.

Lermusiaux, P.F.J., P.J. Haley, Jr., W.G. Leslie, A. Agarwal, O. Logutov and L.J. Burton, 2011. Multiscale Physical and Biological Dynamics in the Philippines Archipelago: Predictions and Processes. Oceanography. PhilEx Issue, 24(1), 70-89, doi:10.5670/oceanog.2011.05.

Lin, Y.-T., A. Newhall, T. F. Duda, P. F. J. Lermusiaux and P. J. H. Haley (2010). "Statistical Merging of Data Sources to Estimate Full WaterColumn Sound Speed in the New Jersey Shelf Shallow Water 2006 Experiment." IEEE Journal of Oceanic Engineering 35(4): 710-721.

Logutov, O. G. and P. F. J. Lermusiaux (2008). "Inverse barotropic tidal estimation for regional ocean applications." Ocean Modelling 25(1-2): 17-34.

Lolla, T. (2012). Path planning in time dependent flows using level set methods. Master's Thesis, Massachusetts Institute of Technology.

Lolla, T., P.F.J. Lermusiaux, M.P. Ueckermann and P.J. Haley, Jr., 2014a. Time-Optimal Path Planning in Dynamic Flows using Level Set Equations: Theory and Schemes. Ocean Dynamics, 64, 10:1373-1397. DOI: 10.1007/s10236-014-0757-y.

Lolla, T., P. J. Haley and P. F. J. Lermusiaux (2014b). "Time-optimal path planning in dynamic flows using level set equations: realistic applications." Ocean Dynamics 64: 1399-1417.

Lolla, T., P.J. Haley. Jr. and P.F.J. Lermusiaux, 2015. Path Planning in Multi-scale Ocean Flows: Coordination and Dynamic Obstacles. Ocean Modelling, 94, 46-66. DOI: 10.1016/j.ocemod.2015.07.013.

Lolla, T. (2016). Path Planning and Adaptive Sampling in the Coastal Ocean. Ph.D. Thesis, Massachusetts Institute of Technology, Department of Mechanical Engineering, February 2016.

Lorenz, E. N. and K. A. Emanuel (1998). "Optimal Sites for Supplementary Weather Observations: Simulation with a Small Model." Journal of the Atmospheric Sciences 55(3): 399-414.

Low, K. H., J. M. Dolan and P. Khosla (2013). Information-theoretic approach to efficient adaptive path planning for mobile robotic environmental sensing. 19th International Conference on Automated Planning and Scheduling (ICAPS 2009).

Majumdar, S. J., C. H. Bishop, B. J. Etherton and Z. Toth (2002). "Adaptive Sampling with the Ensemble Transform Kalman Filter. Part II: Field Program Implementation." Monthly Weather Review 130(5): 1356-1369.

Miettinen, K., 2012. Nonlinear multiobjective optimization (Vol. 12). Springer Science \& Business Media

MSEAS Group, (2013). "Multidisciplinary Simulation, Estimation and Assimilation Systems (MSEAS)." from http://mseas.mit.edu/.

NOAA. (2006). "NGDC Coastal Relief Model." from http://www.ngdc.noaa.gov/mgg/coastal/coastal.html.

Ogren P., E. Fiorelli and N. E. Leonard, 2004 "Cooperative control of mobile sensor networks:Adaptive gradient climbing in a distributed environment," in IEEE Transactions on Automatic Control, vol. 49, no. 8, pp. 1292-1302, doi: 10.1109/TAC.2004.832203

Petillo, S., H. Schmidt, P. F. J. Lermusiaux, D. Yoerger and A. Balasuriya (2015). Autonomous and Adaptive Oceanographic Front Tracking on Board Autonomous Underwater Vehicles. OCEANS 2015, Genoa, Italy.

Ramp, S.R., R. E. Davis, N. E. Leonard, I. Shulman, Y. Chao, A. R. Robinson, J. Marsden, P.F.J. Lermusiaux, D. Fratantoni, J. D. Paduan, F. Chavez, F. L. Bahr, S. Liang, W. Leslie, and Z. Li, 2009. Preparing to Predict: The Second Autonomous Ocean Sampling Network (AOSNII) Experiment in the Monterey Bay. Special issue on AOSN-II, Deep Sea Research, Part II, 56, 68-86, doi: 10.1016/j.dsr2.2008.08.013. 
Ramp, S.R., P.F.J. Lermusiaux, I. Shulman, Y. Chao, R.E. Wolf, and F.L. Bahr, 2011. Oceanographic and Atmospheric Conditions on the Continental Shelf North of the Monterey Bay during August 2006. Dynamics of Atmospheres and Oceans, 52, 192-223, doi:10.1016/j.dynatmoce.2011.04.005.

Robinson, A. R., P. F. J. Lermusiaux and N. Q. Sloan (1998). Data Assimilation. The Sea. K. H. Brink and A. R. Robinson, John Wiley \& Sons. 10: 541-593.

Robinson, A.R., J. Sellschopp, W.G. Leslie, A. Alvarez, G. Baldasserini, P.J. Haley, P.F.J. Lermusiaux, C.J. Lozano, E. Nacini, R. Onken, R. Stoner, P. Zanasca, 2003. Forecasting synoptic transients in the Eastern Ligurian Sea. In "Rapid Environmental Assessment", Bovio, E., R. Tyce and H. Schmidt (Editors), SACLANTCEN Conference Proceedings Series CP-46, Saclantcen, La Spezia, Italy.

Roy N., H.-L. Choi, D. Gombos, J. Hansen, J. How, and S. Park, "Adaptive observation strategies for forecast error minimization," in Computational Science - ICCS 2007, ser. Lecture Notes in Computer Science, Y. Shi, G. D. van Albada, J. Dongarra, and P. M. Sloot, Eds.Springer Berlin Heidelberg, 2007, vol. 4487, pp. 1138-1146.Available: http://dx.doi.org/10.1007/978-3-540-72584-8 149

Schofield, O., S. Glenn, J. Orcutt, M. Arrott, M. Meisinger, A. Gangopadhyay, W. Brown, R. Signell, M. Moline, Y. Chao, S. Chien, D. Thompson, A. Balasuriya, P.F.J. Lermusiaux and M. Oliver, 2010. Automated Sensor Networks to Advance Ocean Science. EOS, Vol. 91, No. 39, 28 September 2010Sloan, I., N. Q. (1996). Process Studies and Data-Driven Simulations in the Middle Atlantic Bight. PhD, Harvard University.

Smith, W. H. F. and D. T. Sandwell (1994). "Bathymetric prediction from dense satellite altimetry and sparse shipboard bathymetry." J. Geophysical Review 99: 21803-21824.

Subramani, D. N., Lolla, T., Haley Jr., P. J., Lermusiaux, P. F. J., 2015. A stochastic optimization method for energy-based path planning. In: Ravela, S., Sandu, A. (Eds.), DyDESS 2014. Vol. 8964 of LNCS. Springer, pp. 1-12.

Subramani, D.N. and P.F.J. Lermusiaux, 2016. Energy-optimal Path Planning by Stochastic Dynamically Orthogonal Level-Set Optimization. Ocean Modeling, 100, 57-77. DOI: 10.1016/j.ocemod.2016.01.006

Tang, D., J. N. Moum, J. F. Lynch, P. Abbot, R. Chapman, P. H. Dahl, T. F. Duda, G. Gawarkiewicz, S. Glenn, J. A. Goff, H. Graber, J. Kemp, A. Maffei, J. D. Nash and A. Newhall (2007). "Shallow Water '06: A joint acoustic propagation/nonlinear internal wave physics experiment. ." Oceanography 20(4): 156-167.

Toth, Z. and E. Kalnay (1997). "Ensemble Forecasting at NCEP and the Breeding Method." Monthly Weather Review 125(12): $3297-3319$.

Wang, D., P. F. J. Lermusiaux, P. J. H. Haley, D. Eickstedt, W. G. Leslie and H. Schmidt (2009). "Acoustically Focused Adaptive Sampling and On-board Routing for Marine Rapid Environmental Assessment." Journal of Marine Systems(Special issue "Coastal Processes: Challenges for Monitoring and Prediction").

"WHOI Shallow Water Experiment." from http://acoustics.whoi.edu/sw06/.

Yilmaz, N. (2005). Path Planning of Autonomous Underwater Vehicles for Adaptive Sampling. PhD PhD, Massacheussetts Institute of Technology.

Yilmaz, N.K., C. Evangelinos, P.F.J. Lermusiaux and N. Patrikalakis, 2008. Path Planning of Autonomous Underwater Vehicles for Adaptive Sampling Using Mixed Integer Linear Programming. IEEE Transactions, Journal of Oceanic Engineering, 33 (4), 522-537. doi: 10.1109/JOE.2008.2002105.

Zhang F., D. M. Fratantoni , D.A. Paley , J.M. Lund, and N.E. Leonard, "Control of coordinated patterns for ocean sampling," International Journal of Control, vol. 80, no. 7, pp. 1186-1199, 2007

Zhang, Y., Godin, M.A., Bellingham, J.G. and Ryan, J.P., 2012. Using an autonomous underwater vehicle to track a coastal upwelling front. Oceanic Engineering, IEEE Journal of, 37(3), pp.338-347. 